\title{
A Generalization Of Inverse Marshall-Olkin Family Of Distributions
}

\author{
K. Jayakumar ${ }^{1}$, K. K. Sankaran ${ }^{2 *}$ \\ ${ }^{1}$ Department of Statistics, University of Calicut, Kerala-673635, India. \\ ${ }^{2}$ Sree Narayana College, Alathur, Kerala-678682, India.
}

\begin{abstract}
We introduce a new family of distributions namely inverse truncated discrete Linnik $\mathrm{G}$ family of distributions. This family is a generalization of inverse Marshall-Olkin family of distributions, inverse family of distributions generated through truncated negative binomial distribution and inverse family of distributions generated through truncated discrete Mittag-Leffler distribution. A particular member of the family, inverse truncated negative binomial Weibull distribution is studied in detail. The shape properties of the probability density function and hazard rate, model identifiability, moments, median, mean deviation, entropy, distribution of order statistics, stochastic ordering property, mean residual life function and stress-strength properties of the new generalized inverse Weibull distribution are studied. The unknown parameters of the distribution are estimated using maximum likelihood method, product spacing method and least square method. The existence and uniqueness of the maximum likelihood estimates are proved. Simulation is carried out to illustrate the performance of maximum likelihood estimates of model parameters. An AR(1) minification model with this distribution as marginal is developed. The inverse truncated negative binomial Weibull distribution is fitted to a real data set and it is shown that the distribution is more appropriate for modeling in comparison with some other competitive models.
\end{abstract}

Keywords: Distribution of order statistics, Entropy, Inverse Weibull distribution, Marshall-Olkin family of distributions, Maximum likelihood, Truncated negative binomial distribution, Weibull distribution

* Corresponding author

Email: snsankaran08@gmail.com 


\section{Introduction}

In the last two decades researchers have greater intention toward the inversion of univariate probability models and their applicability under inverse transformation. The inverse distribution is the distribution of the reciprocal of a random variable. Dubey (1970) proposed inverted beta distribution, Voda (1972) studied inverse Rayleigh distribution, Folks and Chhikara (1978) proposed inverse Gaussian distribution, Prakash (2012) studied the inverted exponential model, Sharma et al. (2015) introduced inverse Lindley distribution, Gharib et al. (2017) studied Marshall-Olkin extended inverse Pareto distribution, Al-Fattah et al. (2017) introduced inverted Kumaraswamy distribution and Rana and Muhammad (2018) introduced Marshall-Olin extended inverted Kumaraswamy distribution.

The inverse Weibull (IW) distribution is commonly used in statistical analysis of lifetime or response time data from reliability experiments. For the situations in which empirical studies indicate that the hazard function might be unimodal, the IW distribution would be an appropriate model. Khan et al. (2008) in their theoretical analysis of IW distribution mention that numerous failure characteristics such as wear out periods and infant mortality can be modeled through IW distribution. They mention the wide range of areas in reliability analysis where IW distribution model can be used successfully. The IW model has been derived as a suitable model for describing the degradation phenomena of mechanical components, such as the dynamic components of diesel engines, see, for example, Murthy et al. (2004). Erto and Rapone (1984) showed that IW model provides good fit to survival data such as the time to breakdown of an insulating fluid subject to the action of constant tension. Interpretation of IW in the context of load strength relationship for a component was provided by Calabria and Pulcini (1994). Furthermore, this distribution is one of the most popular distributions in complementary risk problems. Shafiei et al. (2016) mention that IW distribution is an appropriate model for situation where hazard function is unimodal. The Marshall-Olkin IW distribution and its application in the context of reliability analysis is discussed in Okasha et al. (2017). Hassan and Nassar (2018) studied properties and applications of IW generator of distributions. Compounding IW distribution with zero truncated Poisson and geometric distributions are studied by Chakrabarty and Chowdhury (2018).

Adding parameters to a well-established distribution is a time-honored technique for obtaining more flexible new families of distributions. Marshall and Olkin (1997) discussed a method of adding a new parameter to an existing distribution. It includes the baseline distribution as a special case, and gives more flexibility to model various types of data. One of the important properties of this 
family is that Marshall-Olkin family of distributions possess stability property in the sense that if the method is applied twice, it returns to the same distribution. Also this family satisfies geometric extreme stability property.

Marshall and Olkin (1997) started with a parent survival function $\overline{\mathrm{F}}(\mathrm{x})$ and considered a family of survival functions given by

$$
\bar{G}(x)=\frac{\alpha \bar{F}(x)}{F(x)+\alpha \bar{F}(x)}, \alpha>0 x \in \mathbb{R}
$$

They described the motivation for the family of distributions (1) as follows:

Let $\mathrm{X}_{1}, \mathrm{X}_{2}, \ldots$ be a sequence of independent and identically distributed (i.i.d.) random variables with survival function $\overline{\mathrm{F}}(\mathrm{x})$. Let

$$
U_{N}=\min \left(X_{1}, X_{2}, \ldots, X_{N}\right),
$$

where $\mathrm{N}$ is the geometric random variable with probability mass function (pmf) $\mathrm{P}(\mathrm{N}=\mathrm{n})=\alpha(1-\alpha)^{\mathrm{n}-1}$ for $\mathrm{n}=1,2, \ldots$ and $0<\alpha<1$ and independent of $X_{i}$ 's. Then the random variable $U_{N}$ has the survival function given by (1). If $\alpha>$ 1 and $\mathrm{N}$ is a geometric random variable with pmf of the form $\mathrm{P}(\mathrm{N}=\mathrm{n})=$ $\frac{1}{\alpha}\left(1-\frac{1}{\alpha}\right)^{\mathrm{n}-1}, \mathrm{n}=1,2, \ldots$, then the random variable $\mathrm{V}_{\mathrm{N}}=\max \left(\mathrm{X}_{1}, \mathrm{X}_{2}, \ldots, \mathrm{X}_{\mathrm{N}}\right)$ also has the survival function as (1).

In the past, many authors have studied various univariate distributions belonging to the Marshall-Olkin family of distributions. We refer to the paper of Tahir and Nadarajah (2015) for a list of univariate Marshall-Olkin distributions. Besides special distributions, three families of distributions, which generalize Marshall-Olkin family of distributions, have been recently introduced.

First, Nadarajah, Jayakumar and Ristić (2013) proposed a new generalization of the Marshal-Olkin family of distributions, by replacing the geometric distribution of $\mathrm{N}$ in (2), as truncated negative binomial distribution with pmf given by

$$
P(N=n)=\frac{\alpha^{\theta}}{1-\alpha^{\theta}}\left(\begin{array}{c}
\theta+n-1 \\
\theta-1
\end{array}\right)(1-\alpha)^{n}, n=1,2, \ldots,
$$

where $\alpha>0$ and $\theta>0$. The authors showed that the random minimum, $U_{N}=$ $\min \left(X_{1}, X_{2}, \ldots, X_{N}\right)$ has the survival function of the form

$$
\bar{G}(x ; \alpha, \theta)=\frac{\alpha^{\theta}}{1-\alpha^{\theta}}\left[(F(x)+\alpha \bar{F}(x))^{-\theta}-1\right] .
$$

Note that if $\alpha \rightarrow 1$, then $\bar{G}(x ; \alpha, \theta) \rightarrow \bar{F}(x)$. The family of distributions given in (3) is a generalization of Marshall-Olkin family of distributions, in the sense that when $\theta=1$, (3) reduces to (1).

Pillai and Jayakumar (1995) introduced a class of discrete distributions containing geometric and named it as discrete Mittag-Leffler (DML) distribution, since it arises a discrete analogue of the well known continuous Mittag-Leffler distribution introduced by Pillai (1990). In DML distribution, the probability of 
success depends on the trail number where as in geometric, it is constant through out the trails. Hence, the model obtained by replacing geometric minimum by truncated DML minimum of i.i.d random variables, may be more realistic.

Second generalized family of distributions have been introduced by Sankaran and Jayakumar (2016). The authors introduced a family of distributions by replacing the distribution of $N$ in (2) as the discrete Mittag-Leffler distribution, a generalization of geometric distribution whose probability generating function (pgf) is given by

$$
H(s)=\frac{1}{1+c(1-s)^{\beta}}, c>0,0<\beta \leq 1 .
$$

Using truncated discrete Mittag-Leffler distribution, they derived a family of distributions with parameters $\beta$ and $c$ having survival function

$$
\bar{G}(x)=\frac{1-F^{\beta}(x)}{1+c F^{\beta}(x)} .
$$

Note that, the Marshall-Olkin method applied to $F^{\beta}(x)$, the exponentiated form of a parent distribution function $F$, will also gives rise (4). The family of distributions generated using truncated discrete Mittag Leffler distribution can also be considered as a generalization of Marshall-Olkin family of distributions, since it reduces to Marshall-Olkin family, when $\beta=1$ and $c=\frac{1-\alpha}{\alpha}$.

A non negative integer valued random variable is said to be discrete Linnik distributed, if it has the pgf

$$
H(s)=\left\{\begin{array}{c}
\left(\frac{1}{1+c(1-s)^{\beta}}\right)^{\theta} \text { for } 0<\theta<\infty \\
e^{-c(1-s)^{\beta}} \text { for } \theta=\infty .
\end{array}\right.
$$

Third generalized family of distributions introduced by Jayakuamar and Sankaran (2019) using truncated discrete Linnik family of distributions with parameters $\beta, \theta$ and $c$ have the survival function

$$
\bar{G}(x)=\frac{(1+c)^{\theta}-\left[1+c F^{\beta}(x)\right]^{\theta}}{\left[(1+c)^{\theta}-1\right]\left[1+c F^{\beta}(x)\right]^{\theta}} .
$$

In (5), when $\theta=1$ and $\beta \neq 1$, we obtain the survival function of the family of distributions generated using truncated discrete Mittag-Leffler distribution. When $\beta=1$ and $\theta \neq 1$ in (5), we obtain the survival function of the family of distributions generated using truncated negative binomial distribution in (3). Also when $\beta=1$ and $\theta=1$ in (5), we obtain the survival function of Marshall-Olkin scheme, in (1).

In this paper, we introduce a new family of distributions which we named as inverse truncated discrete Linnik $\mathrm{G}$ family of distributions. In particular, we study 
inverse truncated negative binomial Weibull distribution distribution. The new proposed distribution is a generalization of Marshall-Olkin extended inverse Weibull, Marshall-Olkin extended inverse Rayleigh, Marshall-Olkin extended inverse exponential, inverse Weibull, inverse Rayleigh and inverse exponential distribution.

The rest of the paper is organized as follows. In Section 2, we introduce a new family of distributions, namely inverse truncated discrete Linnik $G$ distribution and their different sub models such as inverse family of distributions generated through discrete Mittag-Leffler $G$ family of distributions, inverse family of distributions generated through truncated negative binomial $G$ family of distributions and inverse family of distributions generated through Marshall-Olkin G family of distributions. In particular, we study inverse truncated negative binomial Weibull (GIW) distribution in Section 3. The shape properties of density and hazard function are studied. The model identifiability of the distribution is proved. The GIW distribution is represented as a mixture of inverse Weibull distribution. In Section 4, some structural properties of GIW distribution such as moments and generating function, quantiles, mean deviation, entropy, order statistics, stochastic ordering and mean residual life function are studied. Method of generation of random variate from GIW distribution is also discussed. Estimation of stress-strength parameters are discussed in Section 5. Estimation of the model parameters by three methods- maximum likelihood estimation, method of product spacing, method of least squares are performed in Section 6. The existence and uniqueness of maximum likelihood estimates are also established.Simulation study is also carried out in order to establish the consistency property of the maximum likelihood estimates of our proposed model. An autoregressive minification process with GIW marginals is developed in Section 7. Finally, in Section 8, we present an application of a real data set, which exhibits the performance of GIW compared to twelve well known models. The GIW distribution has least - $\log$ L, AIC, CAIC, BIC, HQIC, $\mathrm{W}^{*}, \mathrm{~A}^{*}, \mathrm{~K}-\mathrm{S}$ statistic and highest $\mathrm{p}$-value for this data set compared to all other models.

\section{Inverse family of distributions generated through truncated}

\section{discrete Linnik distribution}

Let $\mathrm{X} \sim$ truncated discrete Linnik family of distributions with survival function $S($.$) and baseline distribution function F($.$) . Then Y=\frac{1}{X}$ is an inverse truncated discrete Linnik random variable with cumulative distribution function (cdf) $\mathrm{G}(\mathrm{x})$ given by 


$$
\begin{gathered}
G_{y}(x)=P(Y \leq x)=P\left(\frac{1}{X} \leq x\right)=P\left(X \geq \frac{1}{x}\right)=S(1 / x) \\
=\frac{(1+c)^{\theta}-\left[1+c F^{\beta}(1 / x)\right]^{\theta}}{\left[(1+c)^{\theta}-1\right]\left[1+c F^{\beta}(1 / x)\right]^{\theta}}, \beta, \theta, c>0 ; x>0
\end{gathered}
$$

Hence, we obtain a new family of distributions, which we named as inverse family of distributions generated through discrete Linnik $\mathrm{G}$ distribution.

The probability density function (pdf) and the hazard rate function (hrf) of a random variable from the introduced family are respectively,

$$
g(y, \beta, c, \theta)=\frac{\beta \theta c(1+c)^{\theta} y^{-2} f(1 / y) F^{\beta-1}(1 / y)}{\left[(1+c)^{\theta}-1\right]\left[1+c F^{\beta}(1 / y)\right]^{\theta+1}},
$$

and

$$
h(y, \beta, c, \theta)=\frac{\beta \theta c F^{\beta-1}(1 / y) f(1 / y)}{\left[1+c F^{\beta}(1 / y)\right]\left[\left(1+c F^{\beta}(1 / y)\right)^{\theta}-1\right]}
$$

\subsection{Inverse truncated discrete Mittag-Leffler $G$ family of distributions}

In equation (6), when $\theta=1$, the cdf reduces to inverse truncated discrete Mittag-Leffler $G$ family of distributions. Hence the cdf, pdf and hrf of inverse truncated discrete Mittag-Leffler $G$ family of distributions are respectively:

$$
\begin{gathered}
G(y ; \beta, c, 1)=\frac{1-F^{\beta}(1 / y)}{1+c F^{\beta}(1 / y)}, \\
g(y ; \beta, c, 1)=\frac{\beta(1+c) y^{-2} F^{\beta-1}(1 / y) f(1 / y)}{\left[1+c F^{\beta}(1 / y)\right]^{2}},
\end{gathered}
$$

and

$$
h(y ; \beta, c, 1)=\frac{\beta y^{-2} f(1 / y)}{F(1 / y)\left[1+c F^{\beta}(1 / y)\right]} .
$$

\subsection{Inverse truncated negative binomial G family of distributions}

When in equation(6), $\beta=1$ and $c=\frac{1-\alpha}{\alpha}$, the cdf reduces to inverse truncated negative binomial G family of distributions. So the cdf, pdf and hrf of inverse truncated negative binomial $\mathrm{G}$ family of distributions are respectively:

$$
\begin{gathered}
G(y ; 1, \alpha, \theta)=\frac{\alpha^{\theta}}{1-\alpha^{\theta}}\left[[\alpha+(1-\alpha) F(1 / y)]^{-\theta}-1\right], \\
g(y ; 1, \alpha, \theta)=\frac{\alpha^{\theta}(1-\alpha) y^{-2} \theta f(1 / y)}{\left(1-\alpha^{\theta}\right)(\alpha+(1-\alpha) F(1 / y))^{\theta+1}}
\end{gathered}
$$


and

$$
h(y ; 1, \alpha, \theta)=\frac{\alpha^{\theta}(1-\alpha) y^{-2} \theta f(1 / y)}{[\alpha+(1-\alpha) F(1 / y)]^{\theta}-\alpha^{\theta}} .
$$

\subsection{Inverse Marshall-Olkin G family of distributions}

In equation (6), when $\beta=1, \theta=1$ and $c=\frac{1-\alpha}{\alpha}$, the cdf reduces to inverse Marshall-Olkin G family of distributions. So the cdf, pdf and hrf of inverse Marshall-Olkin G family of distributions are respectively:

$$
\begin{gathered}
G(y ; 1, \alpha, 1)=\frac{\alpha \bar{F}(1 / y)}{1-(1-\alpha) \bar{F}(1 / y)}, \\
g(y ; 1, \alpha, 1)=\frac{\alpha y^{-2} f(1 / y)}{[\alpha+(1-\alpha) F(1 / y)]^{2}},
\end{gathered}
$$

and

$$
h(y ; 1, \alpha, 1)=\frac{\alpha y^{-2} f(1 / y)}{F(1 / y)[\alpha+(1-\alpha) F(1 / y)]} .
$$

Also when $\alpha=1$, the inverse Marshall-Olkin G family of distribution reduces to inverse family of distributions.

\section{A new generalization of inverse Weibull distribution}

Now we consider, generalized inverse Weibull distribution generated through inverse truncated negative binomial and Weibull distribution. Negative binomial is a generalization of the geometric, and Poisson distributions is a limiting particular case. The negative binomial distribution with support over the set of all non-negative integers is also a generalization of the Poisson distribution in the sense that it can deduced as a hierarchial model if $\mathrm{X} \sim$ Poisson $(\Lambda)$ with $\Lambda$ being a gamma random variable.

\subsection{Distribution function}

Let $\mathrm{X}$ follows Weibull distribution with parameters $\lambda>0$ and $\beta>0$ having cdf $F(x)=1-e^{-(\lambda x)^{\beta}}$ and pdf $f(x)=\beta \lambda^{\beta} x^{\beta-1} e^{-(\lambda x)^{\beta}}$. Hence from (12), the cdf of the random variable $\mathrm{Y}$ is given by

$$
G(y ; \alpha, \theta, \lambda, \beta)=\frac{\alpha^{\theta}}{1-\alpha^{\theta}}\left[\frac{1-\left[1-(1-\alpha) e^{-\left(\frac{\lambda}{y}\right)^{\beta}}\right]^{\theta}}{\left[1-(1-\alpha) e^{-\left(\frac{\lambda}{y}\right)^{\beta}}\right]^{\theta}}\right]
$$




\subsection{Probability density function}

The pdf of the new distribution is given by

$$
g(y ; \alpha, \theta, \lambda, \beta)=\frac{\alpha^{\theta}(1-\alpha)^{\theta} \theta \beta \lambda^{\beta} y^{-(\beta+1)} e^{-\left(\frac{\lambda}{y}\right)^{\beta}}}{\left(1-\alpha^{\theta}\right)\left[1-(1-\alpha) e^{-\left(\frac{\lambda}{y}\right)^{\beta}}\right]^{\theta+1}}
$$

We refer to this new distribution having cdf (18) as inverted truncated negative binomial Weibull distribution with parameters $\alpha, \theta, \lambda$ and $\beta$. We write it as $\operatorname{GIW}(y ; \alpha, \theta, \lambda, \beta)$.

The graph of $g(y)$ for different values of the parameters is given in Figure 1.

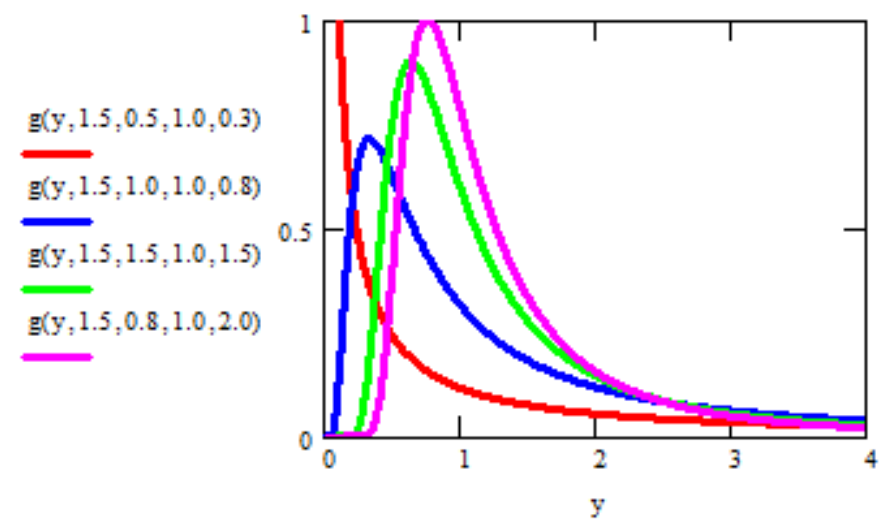

Figure 1: The plots of the pdf of GIW distribution when $\alpha=1.5$ and $\lambda=1.0$.

Some sub-models of the GIW distribution are listed below:

i. When $\theta=1$, we have the inverse Marshall-Olkin Weibull distribution.

ii. When $\theta=1$ and $\beta=2$, we have the inverse Marshall-Olkin Rayleigh distribution.

iii. When $\theta=1$ and $\beta=1$, we have the inverse Marshall-Olkin exponential distribution.

iv. When $\alpha \rightarrow 1$, we have the inverse Weibull distribution.

v. When $\alpha \rightarrow 1$ and $\beta=2$, we have the inverse Rayleigh distribution.

vi. When $\alpha \rightarrow 1$ and $\beta=1$, we have the inverse exponential distribution.

\subsection{Unimodality}

The pdf of the GIW model is either decreasing or unimodal. In order to investigate the critical points of density function, its first derivative with respect to $\mathrm{y}$ is 


$$
\begin{aligned}
g^{\prime}(y) & =\frac{\alpha^{\theta}(1-\alpha)^{\theta} \theta \beta \lambda^{\beta} y^{-(\beta+1)} e^{-\left(\frac{\lambda}{y}\right)^{\beta}}}{\left(1-\alpha^{\theta}\right) y\left[1-(1-\alpha) e^{-\left(\frac{\lambda}{y}\right)^{\beta}}\right]^{\theta+1}}\left[-(\beta+1)+\beta\left(\frac{\lambda}{y}\right)^{\beta}\right. \\
+ & \frac{(1-\alpha) \beta(\theta+1)\left(\frac{\lambda}{y}\right)^{\beta} e^{-\left(\frac{\lambda}{y}\right)^{\beta}}}{\left[1-(1-\alpha) e^{\left.-\left(\frac{\lambda}{y}\right)^{\beta}\right]}\right]}
\end{aligned}
$$

$g^{\prime}(y)=0$ implies,

$$
(1-\alpha) e^{-\left(\frac{\lambda}{y}\right)^{\beta}}\left[(\beta+1)+\theta \beta\left(\frac{\lambda}{y}\right)^{\beta}\right]+\beta\left(\frac{\lambda}{y}\right)^{\beta}=\beta+1
$$

Since equation (20) is a nonlinear equation in $y$, there may be more than one positive root to (20). If $y=y_{0}$ is a root of (20), then it corresponds to a local maximum if $g^{\prime}(y)>0$ for all $y<y_{0}$. It corresponds to a local minimum if $g^{\prime}(y)<0$ for all $y<y_{0}$ and $g^{\prime}(y)>0$ for all $y>y_{0}$. It corresponds to a point of inflexion if either $g^{\prime}(y)>0$ for all $y \neq y_{0}$ or $g^{\prime}(y)<0$ for all $y \neq$ $y_{0}$.

\subsection{Hazard rate}

The hazard rate is given by

$$
h(y)=\frac{\alpha^{\theta}(1-\alpha)^{\theta} \theta \beta \lambda^{\beta} y^{-(\beta+1)} e^{-\left(\frac{\lambda}{y}\right)^{\beta}}}{\left[1-(1-\alpha) e^{-\left(\frac{\lambda}{y}\right)^{\beta}}\right]\left\{\left[1-(1-\alpha) e^{\left.\left.-\left(\frac{\lambda}{y}\right)^{\beta}\right]^{\theta}-\alpha^{\theta}\right\}}\right.\right.} .
$$

The critical point of $h(y)$ is the roots of the equation $\frac{\partial \log h(y)}{\partial y}=0$, which yields

$$
\begin{aligned}
& -\frac{\left(\frac{\lambda}{y}\right)^{\beta}\left(\frac{\beta}{y}\right)}{\left[1-(1-\alpha) e^{-\left(\frac{\lambda}{y}\right)^{\beta}}\right]}\left(\frac{\left[1-(1-\alpha) e^{\left.-\left(\frac{\lambda}{y}\right)^{\beta}\right]^{\theta}}\left[1+\theta(1-\alpha) e^{\left.-\left(\frac{\lambda}{y}\right)^{\beta}\right]-\alpha^{\theta}}\right.\right.}{\left[1-(1-\alpha) e^{\left.-\left(\frac{\lambda}{y}\right)^{\beta}\right]} 3^{\theta}-\alpha^{\theta}\right.}\right) \\
& =0 .
\end{aligned}
$$

There may be more than one roots for (22). Let $\tau(y)=\frac{\partial^{2} \operatorname{logh}(y)}{\partial y^{2}}$. We have $\tau(y)=$ 


$$
\begin{aligned}
& =\frac{\beta+1}{y^{2}}-\frac{2\left(\frac{\lambda}{y^{3}}\right) \beta\left[1-(1-\alpha) e^{\left.-\left(\frac{\lambda}{y}\right)^{\beta}\right]^{\theta-1}\left[1+\theta(1-\alpha) e^{-\left(\frac{\lambda}{y}\right)^{\beta}}-\alpha^{\theta}\right]}\right.}{\left[1-(1-\alpha) e^{\left.-\left(\frac{\lambda}{y}\right)^{\beta}\right]^{\theta}-\alpha^{\theta}}\right.} \\
& +\frac{(1-\alpha) \beta^{2}\left(\frac{\lambda}{y^{3}}\right)\left(\frac{\lambda}{y}\right)^{\beta} e^{-\left(\frac{\lambda}{y}\right)^{\beta}}\left[1-(1-\alpha) e^{\left.-\left(\frac{\lambda}{y}\right)^{\beta}\right]^{\theta-2}\left[1+\theta(1-\alpha) e^{-\left(\frac{\lambda}{y}\right)^{\beta}}-\alpha^{\theta}\right]}\right.}{\left[1-(1-\alpha) e^{\left.-\left(\frac{\lambda}{y}\right)^{\beta}\right]^{\theta}-\alpha^{\theta}}\right.}
\end{aligned}
$$

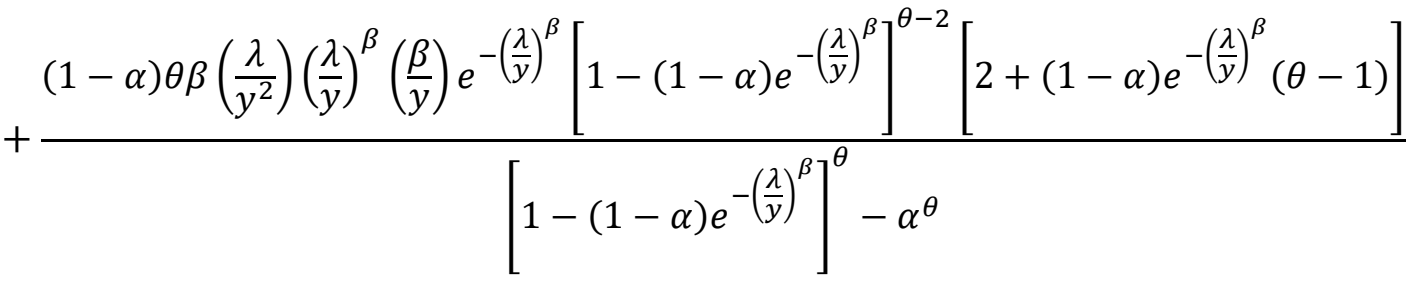

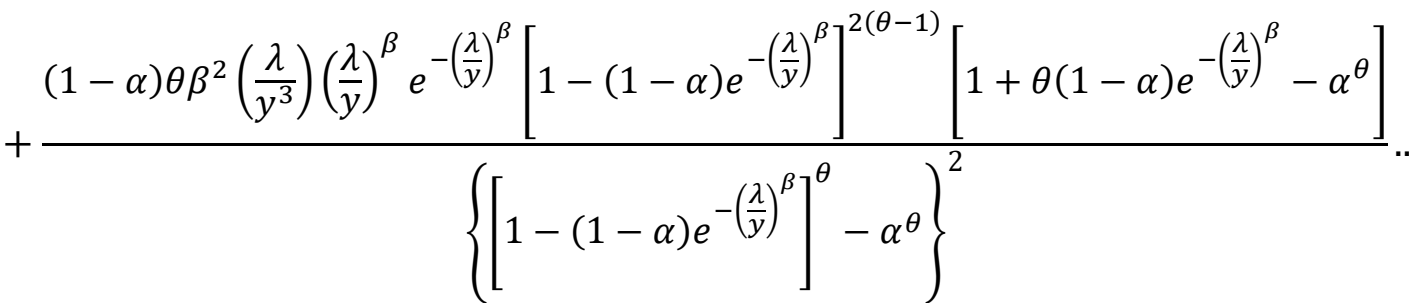

If $y=y_{0}$ is a root of (20), then it refers to a local maximum if $\tau(y)>0$ for all $y<y_{0}$ and $\tau(y)<0$ for all $y>y_{0}$. It corresponds to a local minimum if $\tau(y)<0$ for all $y<y_{0}$ and $\tau(y)>0$ for all $y>y_{0}$. It gives an inflexion point if either $\tau(y)>0$ for all $y \neq y_{0}$ or $\tau(y)<0$ for all $y \neq y_{0}$.

The graph of $\mathrm{h}(\mathrm{y})$ for different values of the parameters is given in Figure 2.

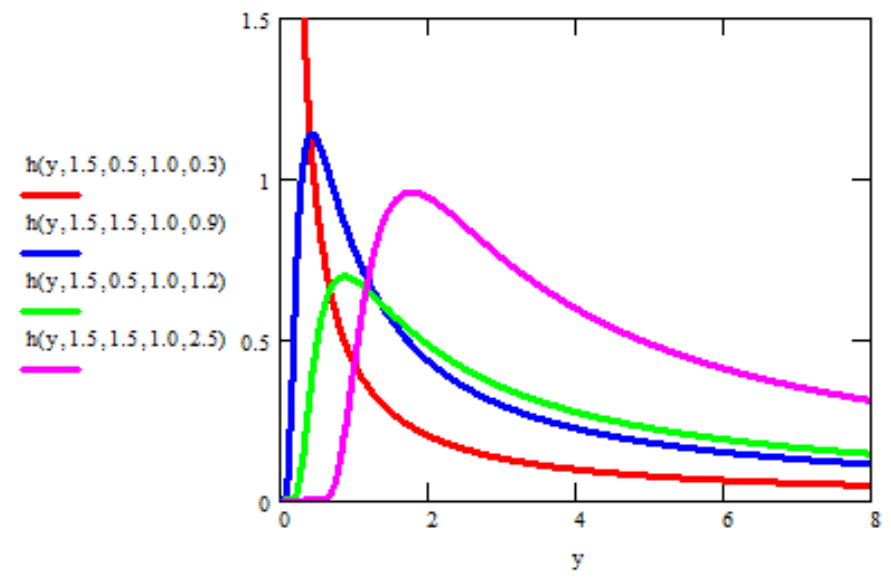

Figure 2: The plots of the hazard rate of GIW distribution when $\alpha=1.5$ and $\lambda=$ 1.0.

Note that inverse truncated negative binomial Weibull (GIW) distribution and truncated negative binomial inverse Weibull distribution are seems to be similar 
structure, but little different. For example, let $G_{1}(y ; \alpha, \theta, \lambda, \beta)$ and $\mathrm{H}_{1}(\mathrm{y} ; \alpha, \theta, \lambda, \beta)$ are the cdf and hazard function of GIW distribution respectively and $G_{2}(y ; \alpha, \theta, \lambda, \beta)$ and $H_{2}(y ; \alpha, \theta, \lambda, \beta)$ are the cdf and hazard function of truncated negative binomial inverse Weibull distribution respectively. In Table 1, we consider the values of cdf and hazard function for $\alpha=1.5, \theta=0.5, \lambda=1.0$ and $\beta=0.5$.

Table 1: The values of cdf and hazard function when $\boldsymbol{\alpha}=\mathbf{1 . 5}, \boldsymbol{\theta}=\mathbf{0 . 5}, \boldsymbol{\lambda}=$ 1.0 and $\beta=0.5$.

\begin{tabular}{|c|c|c|c|c|}
\hline$y$ & $G_{1}(y)$ & $G_{2}(y)$ & $H_{1}(y)$ & $H_{2}(y)$ \\
\hline 0.1 & 0.057 & 0.032 & 0.025 & 0.014 \\
\hline 0.25 & 0.176 & 0.104 & 0.084 & 0.048 \\
\hline 0.5 & 0.304 & 0.192 & 0.157 & 0.093 \\
\hline 1.0 & 0.441 & 0.301 & 0.253 & 0.155 \\
\hline 2.0 & 0.569 & 0.418 & 0.365 & 0.235 \\
\hline 5.0 & 0.706 & 0.567 & 0.531 & 0.363 \\
\hline 10.0 & 0.784 & 0.664 & 0.666 & 0.474 \\
\hline 25.0 & 0.859 & 0.769 & 0.851 & 0.636 \\
\hline 50.0 & 0.899 & 0.829 & 0.995 & 0.766 \\
\hline 100.0 & 0.928 & 0.875 & 1.141 & 0.902 \\
\hline 500.0 & 0.967 & 0.941 & 1.484 & 1.232 \\
\hline 1000.0 & 0.977 & 0.958 & 1.633 & 1.378 \\
\hline
\end{tabular}

\subsection{Model identifiability}

We have to prove model identifiability only with respect to the parameters $\alpha$ and $\theta$, since the other two parameters $(\lambda$ and $\beta)$ are from the parent distribution. Let us suppose that $\mathrm{G}\left(\mathrm{y} ; \alpha_{1}, \theta_{1}\right)=\mathrm{G}\left(\mathrm{y} ; \alpha_{2}, \theta_{2}\right)$ for all $\mathrm{y}>0$. We will show that this condition implies that $\alpha_{1}=\alpha_{2}$ and $\theta_{1}=\theta_{2}$. For proving model identifiability, we use Theorem 2.4 of Chandra (1977).

Proposition : The class of all mixing distribution relative to the GIW distribution is identifiable.

Proof : If $N_{i}$ is truncated negative binomial random variable, truncated at 0 , then the probability generating function is

$$
\phi_{i}(s)=(1-\alpha)^{\theta}\left[\frac{1}{(1-s \alpha)^{\theta}}-1\right] ; i=1,2
$$

From the cdf of $N_{i}$, we have

$G_{1}<G_{2}$ when $\alpha_{1}=\alpha_{2}$ and $\theta_{1}<\theta_{2}$

and 
$G_{1}<G_{2}$ when $\theta_{1}=\theta_{2}$ and $\alpha_{1}<\alpha_{2}$

Let $D_{\phi_{1}(s)}=\left(-\infty, \alpha_{1}\right), D_{\phi_{2}(s)}=\left(-\infty, \alpha_{2}\right)$ and $s=\frac{1}{\alpha_{1}}$. Hence

$$
\lim _{s \rightarrow \frac{1}{\alpha_{1}}} \phi_{1}(s)=\left(1-\alpha_{1}\right)^{\theta_{1}}\left[\frac{1}{\left(1-\frac{1}{\alpha_{1}} \alpha_{1}\right)^{\theta_{1}}}-1\right]=\infty .
$$

When $\theta_{1}=\theta_{2}$ and $\alpha_{2}<\alpha_{1}$, we obtain

$$
\lim _{s \rightarrow \frac{1}{\alpha_{1}}} \phi_{2}(s)=\left(1-\alpha_{2}\right)^{\theta_{1}}\left[\frac{1}{\left(1-\frac{1}{\alpha_{1}} \alpha_{2}\right)^{\theta_{1}}}-1\right]>0 .
$$

So

$$
\lim _{s \rightarrow \alpha_{1}} \frac{\phi_{2}(s)}{\phi_{1}(s)}=0
$$

and thus the identifiability is proved. Hence the $\operatorname{cdf} G$ is identifiable with respect to $\alpha$ and $\theta$.

\subsection{Expansion for distribution function and density function}

For $|\mathrm{z}|<1$ and $\mathrm{k}>0$, we have

$$
(1-\mathrm{z})^{-\mathrm{k}}=\sum_{\mathrm{j}=0}^{\infty} \frac{\Gamma(\mathrm{k}+\mathrm{j})}{\Gamma(\mathrm{k}) \mathrm{j} !} \mathrm{z}^{\mathrm{j}}
$$

where $\Gamma($.$) is the gamma function.$

By using (24), the cdf of GIW distribution can be expressed as

$$
\begin{aligned}
G(y)=\frac{\alpha^{\theta}}{1-\alpha^{\theta}}\left[\left[1-(1-\alpha) e^{-\left(\frac{\lambda}{y}\right)^{\beta}}\right]^{-\theta}-1\right] \\
\quad=\frac{\alpha^{\theta}}{1-\alpha^{\theta}}\left[\sum_{\mathrm{j}=0}^{\infty} \frac{\Gamma(\theta+\mathrm{j})}{\Gamma(\theta) \mathrm{j} !}(1-\alpha)^{\mathrm{j}} e^{-j\left(\frac{\lambda}{y}\right)^{\beta}}-1\right] .
\end{aligned}
$$

In similar manner the pdf of GIW distribution can be expressed as

$$
g(y)=\sum_{\mathrm{j}=0}^{\infty} \frac{\alpha^{\theta}}{1-\alpha^{\theta}} \frac{\Gamma(\theta+\mathrm{j}+1)}{\Gamma(\theta+1) \mathrm{j} !}(1-\alpha)^{\mathrm{j}+1} \theta \beta \lambda^{\beta} y^{-(\beta+1)} e^{-(j+1)\left(\frac{\lambda}{y}\right)^{\beta}}
$$




\section{General properties of GIW distribution}

\subsection{The moments}

We know that moments are important in any statistical analysis. In this subsection, we present $r^{\text {th }}$ moments of GIW distribution.

From the definition of moments, we have

$$
\begin{aligned}
E\left(Y^{r}\right)=\int_{0}^{\infty} y^{r} & \sum_{\mathrm{j}=0}^{\infty} \frac{\alpha^{\theta}}{1-\alpha^{\theta}} \frac{\Gamma(\theta+\mathrm{j}+1)}{\Gamma(\theta+1) \mathrm{j} !}(1-\alpha)^{\mathrm{j}+1} \theta \beta \lambda^{\beta} y^{-(\beta+1)} e^{-(j+1)\left(\frac{\lambda}{y}\right)^{\beta}} d y \\
& =\sum_{\mathrm{j}=0}^{\infty} \frac{\alpha^{\theta}}{1-\alpha^{\theta}} \frac{\Gamma(\theta+\mathrm{j}+1)}{\Gamma(\theta+1) \mathrm{j} !}(1-\alpha)^{\mathrm{j}+1} \theta \beta \lambda^{\beta} \int_{0}^{\infty} y^{r-(\beta+1)} e^{-(j+1)\left(\frac{\lambda}{y}\right)^{\beta}} d y
\end{aligned}
$$

Put $x=\lambda^{\beta}(j+1) y^{-\beta}$. Then,

$$
\begin{gathered}
E\left(Y^{r}\right)=\sum_{\mathrm{j}=0}^{\infty} \frac{\alpha^{\theta}}{1-\alpha^{\theta}} \frac{\Gamma(\theta+\mathrm{j}+1)}{\Gamma(\theta+1)(\mathrm{j}+1) !} \theta(1-\alpha)^{\mathrm{j}+1} \lambda^{r}(j+1)^{\frac{r}{\beta}} \int_{0}^{\infty} x^{\frac{r}{\beta}} e^{-x} d x \\
=\sum_{\substack{\mathrm{j}=0 \\
\beta}} \frac{\alpha^{\theta}}{1-\alpha^{\theta}} \frac{\Gamma(\theta+\mathrm{j}+1)}{\Gamma(\theta+1)(\mathrm{j}+1) !} \theta(1-\alpha)^{\mathrm{j}+1} \lambda^{r}(j+1)^{\frac{r}{\beta}} \Gamma\left(1-\frac{r}{\beta}\right),
\end{gathered}
$$

By putting $r=1$ and $r=2$ in (27), we can easily obtain the mean and variance of GIW distribution.

\subsection{The moment generating function}

The moment generating function is given by

$$
M_{Y}(t)=\int_{0}^{\infty} e^{t y} g(y) d y
$$

By using the Tayler's series of expansion of the function $e^{t y}$, we obtain, 


$$
\begin{aligned}
M_{Y}(t)=\int_{0}^{\infty} \sum_{k=0}^{\infty} \frac{(t y)^{k}}{k !}\left[\sum_{\mathrm{j}=0}^{\infty} \frac{\alpha^{\theta}}{1-\alpha^{\theta}} \frac{\Gamma(\theta+\mathrm{j}+1)}{\Gamma(\theta+1)(\mathrm{j}+1) !}(1\right. \\
-\alpha)^{\mathrm{j}+1} \theta \beta \lambda^{\beta} y^{-(\beta+1)} e^{\left.-(j+1)\left(\frac{\lambda}{y}\right)^{\beta}\right] d y} \\
=\sum_{k=0}^{\infty} \sum_{\mathrm{j}=0}^{\infty} \frac{t^{k}}{k !} \frac{\alpha^{\theta}}{1-\alpha^{\theta}} \frac{\Gamma(\theta+\mathrm{j}+1)}{\Gamma(\theta+1)(\mathrm{j}+1) !}(1 \\
-\alpha)^{\mathrm{j}+1} \theta \beta \lambda^{\beta} \int_{0}^{\infty} y^{k-(\beta+1)} e^{-(j+1)\left(\frac{\lambda}{y}\right)^{\beta}} d y
\end{aligned}
$$

As before, putting $x=\lambda^{\beta}(j+1) y^{-\beta}$ and simplifying, we have

$$
M_{Y}(t)=\sum_{k=0}^{\infty} \sum_{\mathrm{j}=0}^{\infty} \frac{t^{k}}{k !} \frac{\alpha^{\theta}}{1-\alpha^{\theta}} \frac{\Gamma(\theta+\mathrm{j}+1)}{\Gamma(\theta+1)(\mathrm{j}+1) !} \theta(1-\alpha)^{\mathrm{j}+1} \lambda^{k}(j+1)^{\frac{k}{\beta}} \Gamma\left(1-\frac{k}{\beta}\right), \beta>k
$$

\subsection{Simulation and Quantiles}

Random variable $Y$ having GIW distribution can be easily simulated by inverting the cdf. Let $U$ has unform $U(0,1)$ distribution, then

$$
\frac{\alpha^{\theta}}{1-\alpha^{\theta}}\left[\left[1-(1-\alpha) e^{-\left(\frac{\lambda}{y}\right)^{\beta}}\right]^{-\theta}-1\right]=U
$$

which yields

$$
Y=\lambda\left\{-\log \left[\frac{1}{1-\alpha}\left(1-\left[\frac{\alpha^{\theta}}{U\left(1-\alpha^{\theta}\right)+\alpha^{\theta}}\right]^{\frac{1}{\theta}}\right)\right]\right\}^{-\frac{1}{\beta}}
$$

In addition, the $q^{\text {th }}$ quantile $y_{q}$ of GIW distribution is given by

$$
y_{q}=\lambda\left\{-\log \left[\frac{1}{1-\alpha}\left(1-\left[\frac{\alpha^{\theta}}{q\left(1-\alpha^{\theta}\right)+\alpha^{\theta}}\right]^{\frac{1}{\theta}}\right)\right]\right\}^{-\frac{1}{\beta}},
$$

$0<q<1$.

In particular, the median of GIW distribution is given by 


$$
\text { Median }=\lambda\left\{-\log \left[\frac{1}{1-\alpha}\left(1-\left[\frac{\alpha^{\theta}}{0.5\left(1-\alpha^{\theta}\right)+\alpha^{\theta}}\right]^{\frac{1}{\theta}}\right)\right]\right\}^{-\frac{1}{\beta}}
$$

The Bowley's skewness is based on quantiles:

$$
S=\frac{Q(3 / 4)+Q(1 / 4)-2 Q(1 / 2)}{Q(3 / 4)-Q(1 / 4)}
$$

and the Moors' kurtosis is based on octiles:

$$
K=\frac{Q(7 / 8)-Q(5 / 8)+Q(3 / 8)-Q(1 / 8)}{Q(6 / 8)-Q(2 / 8)},
$$

where $Q($.$) represents the quantile function of X$. These measures are less sensitive to outliers and they exist even for distributions without moments. Skewness measures the degree of the long tail and kurtosis is a measure of the degree of peakedness. When the distribution is symmetric, $S=0$ and when the distribution is left(or right) skewed, $S<0$ (or $S>0$ ). As $K$ increases, the tail of the distribution becomes heavier. We compute mean, median, variance, skewness and kurtosis numerically using $\mathrm{R}$ software and presented in Table 2 when $\lambda=1.0$ and $\beta=5.0$.

Table 2: Mean, Median, Variance, Skewness and Kurtosis of GIW distribution for some parameter values when $\lambda=\mathbf{1 . 0}$ and $\boldsymbol{\beta}=\mathbf{5 . 0}$.

\begin{tabular}{|c|l|l|c|c|c|c|}
\hline & & Mean & Median & Variance & Skewness & Kurtosis \\
\hline & $\begin{array}{l}\theta \\
=0.1\end{array}$ & 1.446 & 1.324 & 0.295 & 0.166 & 1.303 \\
\cline { 2 - 7 } $\begin{array}{l}\theta \\
=0.2\end{array}$ & 1.476 & 1.354 & 0.311 & 0.159 & 1.301 \\
\cline { 2 - 7 } & $\begin{array}{l}\theta \\
=0.1\end{array}$ & 1.570 & 1.448 & 0.355 & 0.141 & 1306 \\
\cline { 2 - 7 } & $\begin{array}{l}\theta \\
=1.0\end{array}$ & 1.724 & 1.600 & 0.417 & 0.130 & 1.328 \\
\cline { 2 - 7 } & $\begin{array}{l}\theta \\
=2.0\end{array}$ & 1.987 & 1.849 & 0.501 & 0.143 & 1.352 \\
\cline { 2 - 7 } & $\begin{array}{l}\theta \\
=5.0\end{array}$ & 2.447 & 2.268 & 0.655 & 0.173 & 1.360 \\
\hline \multirow{2}{*}{$\alpha=0.5$} & $\begin{array}{l}\theta \\
=0.1\end{array}$ & 1.235 & 1.139 & 0.166 & 0.185 & 1.348 \\
\cline { 2 - 7 } & $\begin{array}{l}\theta \\
=0.2\end{array}$ & 1.242 & 1.146 & 0.169 & 0.184 & 1.346 \\
\hline
\end{tabular}


16

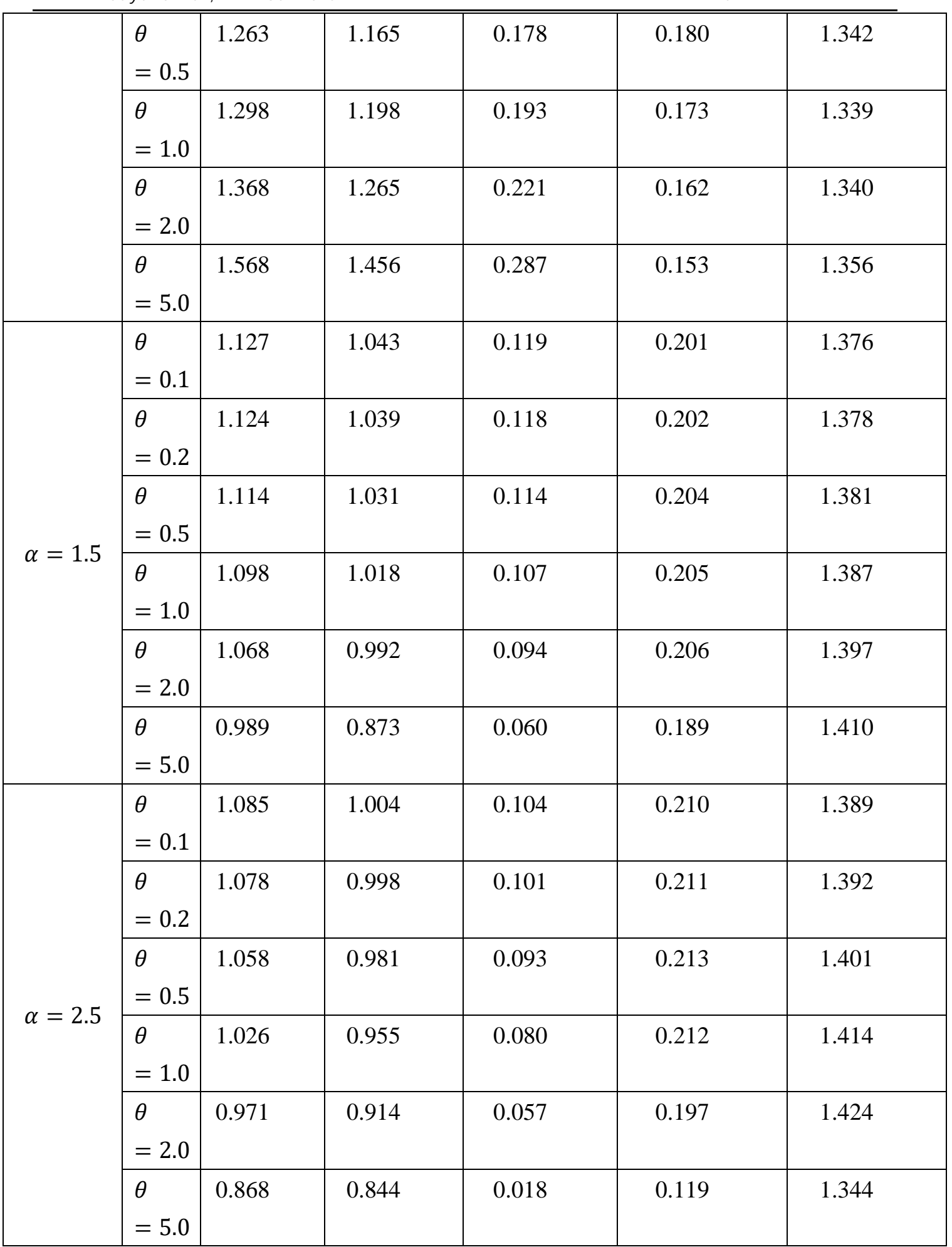

From Table 2, we can see that GIW distribution is positively skewed and leptokurtic. When $\alpha<1$ and $\theta$ is increasing, mean, median and variance are increasing while when $\alpha>1$ and $\theta$ is increasing, mean, median and variance are decreasing.

\subsection{Mean deviation}

The mean deviation about mean is defined by 


$$
\mathrm{D}_{1}(\mathrm{y})=\int_{0}^{\infty}|y-\mu| g(y) d y
$$

where $\mu$ is the mean, which can be rewritten as

$$
\mathrm{D}_{1}(\mathrm{y})=\int_{0}^{\mu}(\mu-y) g(y) d y+\int_{\mu}^{\infty}(y-\mu) g(y) d y .
$$

Using integration by parts, it simplifies to

$$
\mathrm{D}_{1}(\mathrm{y})=2 \mu \mathrm{G}(\mu)-2 \int_{\mu}^{\infty} y g(y) d y,
$$

where G(.) denote the cdf of GIW distribution. Hence,

$$
\mathrm{D}_{1}(\mathrm{y})=2 \mu \mathrm{G}(\mu)-2 \frac{\alpha^{\theta} \theta \lambda}{1-\alpha^{\theta}} \sum_{\mathrm{j}=0}^{\infty} \frac{\Gamma(\theta+\mathrm{j}+1)}{\Gamma(\theta+1)(\mathrm{j}+1) !}(1-\alpha)^{\mathrm{j}+1}(j+1)^{\frac{1}{\beta}} \Gamma\left(1-\frac{1}{\beta}, \mu\right), \beta>1,
$$

where $\Gamma(\mathrm{s}, \mathrm{y})=\int_{\mathrm{y}}^{\infty} t^{s-1} e^{-t} d t, c>0$.

The mean deviation about median is defined as

$$
\mathrm{D}_{2}(\mathrm{y})=\int_{0}^{\infty}|y-M| g(y) d y=\int_{0}^{\mathrm{M}}(M-y) g(y) d y+\int_{\mathrm{M}}^{\infty}(y-M) g(y) d y,
$$

where M stands for median. Hence,

$\mathrm{D}_{2}(\mathrm{y})=-\mu+2 \int_{M}^{\infty} y g(y) d y$

$$
=-\mu+2 \frac{\alpha^{\theta} \theta \lambda}{1-\alpha^{\theta}} \sum_{\mathrm{j}=0}^{\infty} \frac{\Gamma(\theta+\mathrm{j}+1)}{\Gamma(\theta+1)(\mathrm{j}+1) !}(1-\alpha)^{\mathrm{j}+1}(j+1)^{\frac{1}{\beta}} \Gamma\left(1-\frac{1}{\beta}, \mathrm{M}\right), \beta>1 .
$$

\subsection{Entropy}

An entropy is a measure of variation or uncertainty. The Rényi entropy of a random variable with pdf $\mathrm{g}($.$) is defined as$

$$
\mathrm{I}_{\mathrm{R}}(\gamma)=\frac{1}{1-\gamma} \log \int_{0}^{\infty} \mathrm{g}^{\gamma}(\mathrm{y}) \mathrm{dy}, \gamma>0, \gamma \neq 1
$$

We have

$$
\begin{aligned}
\int_{0}^{\infty} \mathrm{g}^{\gamma}(\mathrm{y}) \mathrm{dy}= & {\left[\frac{\alpha^{\theta}(1-\alpha) \theta \beta \lambda^{\beta}}{1-\alpha^{\theta}}\right]^{\gamma} \int_{0}^{\infty} \frac{\mathrm{y}^{-\gamma(\beta+1)} e^{-\gamma\left(\frac{\lambda}{y}\right)^{\beta}}}{\left[1-(1-\alpha) e^{-\left(\frac{\gamma}{y}\right)}\right]^{\gamma(\theta+1)}} \mathrm{dy} } \\
& =\left[\frac{\alpha^{\theta}(1-\alpha) \theta \beta \lambda^{\beta}}{1-\alpha^{\theta}}\right]^{\gamma} \frac{1}{\left[\lambda^{\beta} j(\gamma+1)\right]^{\frac{(\beta+1)(\gamma-1)}{\beta}}} \Gamma\left[\frac{\gamma(1+\beta)-1}{\beta}\right] .
\end{aligned}
$$


Therefore,

$$
\begin{aligned}
\mathrm{I}_{\mathrm{R}}(\gamma)=\frac{\gamma}{1-\gamma} \log \left[\frac{\alpha^{\theta}(1-\alpha) \theta \beta \lambda^{\beta}}{1-\alpha^{\theta}}\right]-\frac{(\beta+1)(\gamma-1)}{\beta(\gamma-1)} \log \left[\gamma^{\beta} \mathrm{j}(\gamma+1)\right] \\
+\frac{1}{1-\gamma} \log \left(\Gamma\left[\frac{\gamma(1+\beta)-1}{\beta}\right]\right) .
\end{aligned}
$$

\subsection{Order statistics}

Let $Y_{1}, Y_{2}, \ldots, Y_{n}$ be a random sample of size $n$ from the GIW distribution and $Y_{(1)}, Y_{(2)}, \ldots, Y_{(n)}$ denote the corresponding order statistics. When the population cdf and pdf are $G(y)$ and $g(y)$ respectively, then the $r^{\text {th }}$ order $(r=$ $1,2, \ldots, n)$ cdf and pdf are respectively, given by,

$$
\begin{aligned}
\mathrm{G}_{(\mathrm{r})}(\mathrm{y})=\sum_{\mathrm{c}=\mathrm{r}}^{\mathrm{n}}\left(\begin{array}{l}
n \\
c
\end{array}\right) G^{c}(y)[1-G(y)]^{n-c}=\sum_{\mathrm{c}=\mathrm{r}}^{\mathrm{n}} \sum_{\mathrm{d}=0}^{\mathrm{n}-\mathrm{c}}\left(\begin{array}{l}
n \\
c
\end{array}\right)\left(\begin{array}{c}
n-c \\
d
\end{array}\right)(-1)^{d} G^{c+d}(y) \\
=\sum_{\mathrm{c}=\mathrm{r}}^{n} \sum_{\mathrm{d}=0}^{\mathrm{n}-\mathrm{c}}\left(\begin{array}{l}
n \\
c
\end{array}\right)\left(\begin{array}{c}
n-c \\
d
\end{array}\right)(-1)^{d}\left[\frac{\alpha^{\theta}}{1-\alpha^{\theta}}\right]^{c+d}\left\{\left[1-(1-\alpha) e^{-\left(\frac{\lambda}{y}\right)^{\beta}}\right]^{-\theta}-1\right\}^{c+d},
\end{aligned}
$$

and

$$
\begin{aligned}
& \mathrm{g}_{(\mathrm{r})}(\mathrm{y})=\frac{n !}{(r-1) !(n-r) !} G^{r-1}(y)[1-G(y)]^{n-r} g(y) \\
& =\frac{n !}{(r-1) !(n-r) !} \sum_{d=0}^{n-r}(-1)^{d}\left(\begin{array}{c}
n-r \\
d
\end{array}\right) G^{r+d-1}(y) g(y) \\
& =\frac{n !}{(r-1) !(n-r) !} \sum_{d=0}^{n-r}(-1)^{d}\left(\begin{array}{c}
n-r \\
d
\end{array}\right)(1 \\
& -\alpha) \theta \beta \lambda^{\theta} y^{-(\beta+1)} e^{-\left(\frac{\lambda}{y}\right)^{\beta}}\left[\frac{\alpha^{\theta}}{1-\alpha^{\theta}}\right]^{r+d} \frac{\left\{1-\left[1-(1-\alpha) e^{-\left(\frac{\lambda}{y}\right)^{\beta}}\right]^{\theta}\right\}^{r+d-1}}{\left[1-(1-\alpha) e^{-\left(\frac{\lambda}{y}\right)^{\beta}}\right]^{\theta(r+d)+1}} .
\end{aligned}
$$

Define the minimum as $\mathrm{Y}_{(1)}=\min \left(\mathrm{Y}_{1}, \mathrm{Y}_{2}, \ldots, \mathrm{Y}_{\mathrm{n}}\right)$, the maximum as $\mathrm{Y}_{(\mathrm{n})}=$ $\max \left(Y_{1}, Y_{2}, \ldots, Y_{n}\right)$ and the median as $Y_{(m+1)}$ with $m=[n / 2]$. Therefore the pdf of the minimum, maximum and median are respectively

$$
\begin{aligned}
& \mathrm{g}_{(1)}(\mathrm{y})=n[1-G(y)]^{n-1} g(y) \\
& =\frac{n \alpha^{\theta}(1-\alpha) \theta \beta \lambda^{\theta} y^{-(\beta+1)} e^{-\left(\frac{\lambda}{y}\right)^{\beta}}\left\{\left[1-(1-\alpha) e^{\left.\left.-\left(\frac{\lambda}{y}\right)^{\beta}\right]^{\theta}-\alpha^{\theta}\right\}^{n-1}}\right.\right.}{\left(1-\alpha^{\theta}\right)^{n}\left[1-(1-\alpha) e^{-\left(\frac{\lambda}{y}\right)^{\beta}}\right]^{n \theta+1}} .
\end{aligned}
$$




$$
\begin{aligned}
& \mathrm{g}_{(\mathrm{n})}(\mathrm{y})=n[G(y)]^{n-1} g(y) \\
& =n\left[\frac{\alpha^{\theta}}{1-\alpha^{\theta}}\right]^{n} \frac{(1-\alpha) \theta \beta \lambda^{\theta} y^{-(\beta+1)} e^{-\left(\frac{\lambda}{y}\right)^{\beta}}\left\{1-\left[1-(1-\alpha) e^{-\left(\frac{\lambda}{y}\right)^{\beta}}\right]^{\theta}\right\}^{n-1}}{\left[1-(1-\alpha) e^{-\left(\frac{\lambda}{y}\right)^{\beta}}\right]^{n \theta+1}} . \\
& \mathrm{g}_{(\mathrm{m}+1)}(\mathrm{y})=\frac{(2 m+1) !}{m ! m !}[G(y)]^{m}[1-G(y)]^{m} g(y) \\
& =\frac{(2 m+1) !}{(m !)^{2}} \frac{\alpha^{m \theta+1}\left\{1-\left[1-(1-\alpha) e^{\left.\left.-\left(\frac{\lambda}{y}\right)^{\beta}\right]^{\theta}\right\}^{m}\left\{\left[1-(1-\alpha) e^{-\left(\frac{\lambda}{y}\right)^{\beta}}\right]^{\theta}-\alpha^{\theta}\right\}}\right.\right.}{\left(1-\alpha^{\theta}\right)^{2 m+1}\left[1-(1-\alpha) e^{-\left(\frac{\lambda}{y}\right)^{\beta}}\right]^{\theta(2 m+1)+1}}(1 \\
& -\alpha) \theta \beta \lambda^{\theta} y^{-(\beta+1)} e^{-\left(\frac{\lambda}{y}\right)^{\beta}} \text {. }
\end{aligned}
$$

\subsection{Stochastic ordering}

Stochastic orders have been used during the last forty years, at an accelerated rate, in many diverse areas of probability and statistics. Such areas include reliability theory, survival analysis, queueing theory, biology, economics, insurance and actuarial science (see, Shaked and Shanthikumar (2007)). Let X and $\mathrm{Y}$ be two random variables having cdf's $\mathrm{F}$ and $\mathrm{G}$ respectively, and denote by $\bar{F}=1-F$ and $\bar{G}=1-G$ their respective survival functions, with corresponding pdf's $\mathrm{f}, \mathrm{g}$. The random variable $\mathrm{X}$ is said to be smaller than $\mathrm{Y}$ in the:

i. $\quad$ stochastic order (denoted as $\mathrm{X} \leq_{\text {st }} \mathrm{Y}$ ) if $\overline{\mathrm{F}}(x) \leq \overline{\mathrm{G}}(x)$ for all $\mathrm{x}$;

ii. likelihood ratio order (denoted as $\mathrm{X} \leq_{\operatorname{lr}} \mathrm{Y}$ ) if $\mathrm{f}(\mathrm{x}) / \mathrm{g}(\mathrm{x})$ is decreasing in $\mathrm{x} \geq 0$;

iii. hazard rate order (denoted as $\mathrm{X} \leq_{\mathrm{hr}} \mathrm{Y}$ )if $\overline{\mathrm{F}}(x) / \overline{\mathrm{G}}(x)$ is decreasing in $\mathrm{x} \geq$ 0 ;

iv. reversed hazard rate order (denoted as $\mathrm{X} \leq_{\mathrm{rhr}} \mathrm{Y}$ ) if $\mathrm{F}(\mathrm{x}) / \mathrm{G}(\mathrm{x})$ is decreasing in $\mathrm{x} \geq 0$.

The four stochastic orders defined above are related to each other, have the following implications (see, Shaked and Shanthikumar (2007)):

$$
\mathrm{X} \leq_{\mathrm{rhr}} \mathrm{Y} \Leftarrow \mathrm{X} \leq_{\mathrm{lr}} \mathrm{Y} \Rightarrow \mathrm{X} \leq_{\mathrm{hr}} \mathrm{Y} \Rightarrow \mathrm{X} \leq_{\mathrm{st}} \mathrm{Y} .
$$

\section{Case I : $\boldsymbol{\theta}_{1}<\boldsymbol{\theta}_{2}$}

Let $\mathrm{X} \sim \operatorname{GIW}\left(\alpha, \theta_{1}, \lambda, \beta\right)$ and $\mathrm{Y} \sim \operatorname{GIW}\left(\alpha, \theta_{2}, \lambda, \beta\right)$. Then 


$$
\frac{g_{\mathrm{X}}(y)}{g_{\mathrm{Y}}(y)}=\frac{\alpha_{1}^{\theta} \theta_{1}\left(1-\alpha_{2}^{\theta}\right)\left[1-(1-\alpha) e^{-\left(\frac{\lambda}{y}\right)^{\beta}}\right]^{\theta_{2}+1}}{\alpha_{2}^{\theta} \theta_{2}\left(1-\alpha_{1}^{\theta}\right)\left[1-(1-\alpha) e^{-\left(\frac{\lambda}{y}\right)^{\beta}}\right]^{\theta_{1}+1}} .
$$

Since $\theta_{1}<\theta_{2}$,

$$
\begin{aligned}
\frac{d}{d x}\left[\frac{g_{\mathrm{X}}(y)}{g_{\mathrm{Y}}(y)}\right]= & -\alpha^{\frac{\theta_{1}}{\theta_{2}}} \frac{\theta_{1}\left(1-\alpha_{2}^{\theta}\right)}{\theta_{2}\left(1-\alpha_{1}^{\theta}\right)}\left[\theta_{2}\right. \\
& \left.-\theta_{1}\right)\left(\frac{\lambda}{y}\right)^{\beta}\left(\frac{\beta}{y}\right) e^{-\left(\frac{\lambda}{y}\right)^{\beta}}\left[1-(1-\alpha) e^{\left.-\left(\frac{\lambda}{y}\right)^{\beta}\right]^{\theta_{2}-\theta_{1}-1}}\right]<0 .
\end{aligned}
$$

Hence, $g_{X}(y) / g_{Y}(y)$ is decreasing in $y$. That is $\mathrm{X} \leq_{\operatorname{lr}} \mathrm{Y}$. The remaining statement follow from the implication (32).

\section{Case II : $\alpha_{2}<\alpha_{1}$}

Let $\mathrm{X} \sim \operatorname{GIW}\left(\alpha_{1}, \theta, \lambda, \beta\right)$ and $\mathrm{Y} \sim \operatorname{GIW}\left(\alpha_{2}, \theta, \lambda, \beta\right)$. Then

$$
\frac{g_{\mathrm{X}}(y)}{g_{\mathrm{Y}}(y)}=\frac{\alpha_{1}^{\theta}\left(1-\alpha_{1}\right)\left(1-\alpha_{2}^{\theta}\right)}{\alpha_{2}^{\theta}\left(1-\alpha_{2}\right)\left(1-\alpha_{1}^{\theta}\right)}\left[\frac{1-\left(1-\alpha_{2}\right) e^{-\left(\frac{\lambda}{y}\right)^{\beta}}}{1-\left(1-\alpha_{1}\right) e^{-\left(\frac{\lambda}{y}\right)^{\beta}}}\right]^{\theta+1} .
$$

Since $\alpha_{2}<\alpha_{1}$,

$$
\frac{d}{d x}\left[\frac{g_{\mathrm{X}}(y)}{g_{\mathrm{Y}}(y)}\right]=-\left(\alpha_{1}-\alpha_{2}\right)(\theta+1)\left(\frac{\lambda}{y}\right)^{\beta}\left(\frac{\beta}{y}\right) e^{-\left(\frac{\lambda}{y}\right)^{\beta}} \frac{\left[1-\left(1-\alpha_{2}\right) e^{-\left(\frac{\lambda}{y}\right)^{\beta}}\right]^{\theta}}{\left[1-\left(1-\alpha_{1}\right) e^{-\left(\frac{\lambda}{y}\right)^{\beta}}\right]^{\theta+2}}<0 .
$$

Hence, $g_{X}(y) / g_{Y}(y)$ is decreasing in $y$. That is $\mathrm{X} \leq_{\operatorname{lr}} \mathrm{Y}$.. The remaining statement follow from the implication (32).

\subsection{Mean residual life function}

Given that a component survives up to time $t \geq 0$, the residual life is the period beyond $t$ until the time of failure and defined by the conditional random variable $\mathrm{Y}-\mathrm{t} \mid \mathrm{Y}>\mathrm{t}$. The mean residual life (MRL) function is an important function in survival analysis, actuarial science, economics and other social sciences and reliability for characterizing life time. Although the shape of the failure rate function plays an important role in repair and replacement strategies, the MRL function is more relevant as the latter summarize the entire residual life function, where the former considers only the risk of instantaneous failure. In reliability, it is well known that the MRL function and ratio of consecutive moments of residual life determine the distribution uniquely (Gupta and Gupta (1983)). 
The $r^{\text {th }}$ order moment of the residual life time of the distribution is given by the general formula:

$$
m_{r}(t)=E\left[(\mathrm{Y}-\mathrm{t})^{\mathrm{r}} \mid \mathrm{Y}>\mathrm{t}\right]=\frac{1}{\bar{G}(t)} \int_{t}^{\infty}(y-t)^{r} g(y) d y .
$$

Hence the MRL function of the GIW distribution is given by

$$
\begin{aligned}
& m_{1}(t)=\frac{1}{\bar{G}(t)} \int_{t}^{\infty}(y-t) g(y) d y=\frac{1}{\bar{G}(t)} \int_{t}^{\infty} y g(y) d y-t \\
& \quad=\frac{1}{\bar{G}(t)} \sum_{j=0}^{\infty} \frac{\alpha^{\theta} \Gamma(\theta+\mathrm{j}+1)}{\left(1-\alpha^{\theta}\right) \Gamma(\theta+1) \mathrm{j} !}(1-\alpha)^{j+1} \theta \beta \lambda^{\beta} \int_{t}^{\infty} y^{-\beta} e^{-\lambda^{\beta}(j+1) y^{-\beta}} d y-t \\
& \quad=\frac{1}{\bar{G}(t)} \sum_{j=0}^{\infty} \frac{\alpha^{\theta} \Gamma(\theta+\mathrm{j}+1)(1-\alpha)^{j+1} \theta \lambda(j+1)^{\frac{1}{\beta}}}{\left(1-\alpha^{\theta}\right) \Gamma(\theta+1)(\mathrm{j}+1) !} \gamma\left(1-\frac{1}{\beta}, \lambda^{\beta}(j+1) y^{-\beta}\right)-t
\end{aligned}
$$

where $\gamma(\mathrm{c}, \mathrm{x})=\int_{0}^{\mathrm{x}} \mathrm{y}^{\mathrm{c}-1} \mathrm{e}^{-\mathrm{y}} d y, \mathrm{c}>0$. On the other hand, we analogously discuss the reversed residual life and some of its properties. The reversed residual life can be defined as the conditional random variable $t-\mathrm{Y} \mid \mathrm{Y} \leq \mathrm{t}$ which denotes the time elapsed from the failure of a component given that its life is less than or equal to $t$. This random variable may also be called the inactivity time (or time since failure) (see Nanda et al. (2003) and Kundu and Nanda (2010)). Also in reliability, the mean reversed residual life (MRRL) and ratio of two consecutive moments of reversed residual life characterize the distribution uniquely. The reversed hazard rate function of the GIW distribution is given by

$$
r(x)=\frac{f(x)}{F(x)}=\frac{(1-\alpha) \theta \beta \lambda^{\beta} y^{-(\beta+1)} e^{-\left(\frac{\lambda}{y}\right)^{\beta}}}{\left[1-(1-\alpha) e^{-\left(\frac{\lambda}{y}\right)^{\beta}}\right]\left\{1-\left[1-(1-\alpha) e^{-\left(\frac{\lambda}{y}\right)^{\beta}}\right]^{\theta}\right\}}
$$

The $r^{\text {th }}$ moment of the reversed residual life function can be obtained the formula

$$
\mu_{r}(x)=E\left[(t-y)^{r} \mid Y \leq t\right]=\frac{1}{G(t)} \int_{0}^{t}(t-y)^{r} g(y) d y .
$$

Hence the MRRL function of the GIW distribution is given by

$$
\begin{array}{r}
\mu_{1}(x)=\frac{1}{G(t)} \int_{0}^{t}(t-y) g(y) d y=t-\frac{1}{G(t)} \sum_{j=0}^{\infty} \frac{\alpha^{\theta} \Gamma(\theta+\mathrm{j}+1)(1-\alpha)^{j} \theta \beta \lambda^{\beta}}{\left(1-\alpha^{\theta}\right) \Gamma(\theta+1) \mathrm{j} !} \int_{t}^{\infty} y^{-\beta} e^{-\left(\frac{\lambda}{y}\right)^{\beta}} d y \\
=t-\frac{1}{G(t)} \sum_{j=0}^{\infty} \frac{\alpha^{\theta} \Gamma(\theta+\mathrm{j}+1)(1-\alpha)^{j+1} \theta \lambda(j+1)^{\frac{1}{\beta}}}{\left(1-\alpha^{\theta}\right) \Gamma(\theta+1)(\mathrm{j}+1) !} \Gamma\left(\frac{1}{\beta}, \lambda(j+1) t^{-\beta}\right), \beta>1,
\end{array}
$$

where $\Gamma(c, x)=\int_{x}^{\infty} y^{c-1} e^{-y} d y, c>0$. 


\section{Stress-strength parameter}

Suppose that the random variable $\mathrm{Y}$ is the strength of a component, which is subjected to a random stress $\mathrm{Z}$. The component fails whenever $\mathrm{Y}<\mathrm{Z}$ and there is no hazard when $\mathrm{Y}>\mathrm{Z}$. In the context of reliability, the stress-strength parameter $\mathrm{R}=\mathrm{P}(\mathrm{Y}>\mathrm{Z})$ is a measure of component reliability and its estimation when $\mathrm{Y}$ and $\mathrm{Z}$ are independent and follow a specified distribution has been discussed widely in the literature. Here, we estimate $\mathrm{R}=\mathrm{P}(\mathrm{Y}>\mathrm{Z})$ for the GIW distribution.

Case I : $\theta_{1} \neq \theta_{2}$

Assume that $\mathrm{Y} \sim \operatorname{GIW}\left(\alpha, \theta_{1}, \lambda, \beta\right)$ and $\mathrm{Z} \sim \operatorname{GIW}\left(\alpha, \theta_{2}, \lambda, \beta\right)$ are independent. The pdf of $\mathrm{Y}$ and the cdf of $\mathrm{Z}$ can be expressed respectively as:

$$
\begin{gathered}
g_{1}(y)=\sum_{j=0}^{\infty} \frac{\alpha^{\theta_{1}}}{1-\alpha^{\theta_{1}}} \frac{\Gamma\left(\theta_{1}+\mathrm{j}+1\right)}{\Gamma\left(\theta_{1}+1\right) \mathrm{j} !}(1-\alpha)^{j+1} \theta_{1} \beta \lambda^{\beta} y^{-(\beta+1)} e^{-\lambda^{\beta}(j+1) y^{-\beta}}, \\
G_{2}(y)=\frac{\alpha^{\theta_{2}}}{1-\alpha^{\theta_{2}}}\left[\sum_{k=0}^{\infty} \frac{\Gamma\left(\theta_{2}+\mathrm{k}\right)}{\Gamma\left(\theta_{2}\right) \mathrm{k} !}(1-\alpha)^{k} e^{-\lambda^{\beta} k y^{-\beta}}-1\right] .
\end{gathered}
$$

We have

$$
\begin{aligned}
& \mathrm{R}=\int_{0}^{\infty} g_{1}(y) G_{2}(y) d y \\
& =\sum_{j=0}^{\infty} \sum_{k=0}^{\infty} \frac{\alpha^{\theta_{1}+\theta_{2}} \Gamma\left(\theta_{1}+\mathrm{j}+1\right) \Gamma\left(\theta_{2}+\mathrm{k}\right)(1-\alpha)^{j+k+1} \theta_{1} \beta \lambda^{\beta}}{\left(1-\alpha^{\theta_{1}}\right)\left(1-\alpha^{\theta_{2}}\right) \Gamma\left(\theta_{1}+1\right) \Gamma\left(\theta_{2}\right) \mathrm{j} ! \mathrm{k} !} \\
& \int_{0}^{\infty} y^{-(\beta+1)} e^{-\lambda^{\beta}(j+k+1) y^{-\beta}} d y-\frac{\alpha^{\theta_{2}}}{1-\alpha^{\theta_{2}}} \\
& =\sum_{j=0}^{\infty} \sum_{k=0}^{\infty} \frac{\alpha^{\theta_{1}+\theta_{2}} \Gamma\left(\theta_{1}+\mathrm{j}+1\right) \Gamma\left(\theta_{2}+\mathrm{k}\right)(1-\alpha)^{j+k+1} \theta_{1}}{\left(1-\alpha^{\theta_{1}}\right)\left(1-\alpha^{\theta_{2}}\right) \Gamma\left(\theta_{1}+1\right) \Gamma\left(\theta_{2}\right) \mathrm{j} ! \mathrm{k} !(\mathrm{j}+\mathrm{k}+1)}-\frac{\alpha^{\theta_{2}}}{1-\alpha^{\theta_{2}} .}
\end{aligned}
$$

Let us assume that $\mathrm{y}_{1}, \mathrm{y}_{2}, \ldots, \mathrm{y}_{\mathrm{n}}$ and $\mathrm{z}_{1}, \mathrm{z}_{2}, \ldots, \mathrm{z}_{\mathrm{m}}$ are independent observations from $\mathrm{Y}$ and $\mathrm{Z}$ respectively. The total log-likelihood function $\mathrm{L}_{\mathrm{R}}\left(\Theta^{*}\right)$ where $\Theta^{*}=\left(\alpha, \theta_{1}, \theta_{2}, \lambda, \beta\right)^{\mathrm{T}}$, becomes 


$$
\begin{aligned}
\mathrm{L}_{\mathrm{R}}\left(\Theta^{*}\right)=\log ( & \alpha)\left(n \theta_{1}+m \theta_{2}\right)-n \log \left(1-\alpha^{\theta_{1}}\right)-m \log \left(1-\alpha^{\theta_{2}}\right)+n \log \left(\theta_{1}\right) \\
+ & m \log \left(\theta_{2}\right)+(n+m) \log \left(\beta \lambda^{\beta}\right)-(\beta+1)\left[\sum_{i=1}^{n} \log \left(y_{i}\right)+\sum_{j=1}^{m} \log \left(z_{i}\right)\right] \\
& -\sum_{i=1}^{n}\left(\frac{\lambda}{\mathrm{y}_{\mathrm{i}}}\right)^{\beta}-\sum_{j=1}^{m}\left(\frac{\lambda}{\mathrm{z}_{\mathrm{j}}}\right)^{\beta}-\left(\theta_{1}+1\right) \sum_{i=1}^{n} \log \left[1-(1-\alpha) e^{\left.-\left(\frac{\lambda}{y_{i}}\right)^{\beta}\right]}\right. \\
& -\left(\theta_{2}+1\right) \sum_{j=1}^{m} \log \left[1-(1-\alpha) e^{\left.-\left(\frac{\lambda}{z_{j}}\right)^{\beta}\right] .}\right.
\end{aligned}
$$

By taking the first partial derivatives of the total log-likelihood function with respect to five parameters in $\Theta^{*}$, we obtain the five normal equations. The maximum likelihood estimates $\widehat{\Theta}^{*}$ of $\Theta^{*}$ is obtained by solving the system of non linear normal equations numerically. From the solution of these equations, we can estimate $\mathrm{R}$ by inserting the estimate of $\widehat{\Theta}^{*}$ in equation (33).

\section{Case II : $\alpha_{1} \neq \alpha_{2}, \theta_{1} \neq \theta_{2}$}

Assume that $\mathrm{Y} \sim \operatorname{GIW}\left(\alpha_{1}, \theta_{1}, \lambda, \beta\right)$ and $\mathrm{Z} \sim \operatorname{GIW}\left(\alpha_{2}, \theta_{2}, \lambda, \beta\right)$ are independent. The pdf of $\mathrm{Y}$ and the cdf of $\mathrm{Z}$ can be expressed respectively as:

$$
\begin{gathered}
g_{1}(y)=\sum_{j=0}^{\infty} \frac{\alpha_{1}^{\theta_{1}}}{1-\alpha_{1}^{\theta_{1}}} \frac{\Gamma\left(\theta_{1}+\mathrm{j}+1\right)}{\Gamma\left(\theta_{1}+1\right) \mathrm{j} !}\left(1-\alpha_{1}\right)^{j+1} \theta_{1} \beta \lambda^{\beta} y^{-(\beta+1)} e^{-\lambda^{\beta}(j+1) y^{-\beta}}, \\
G_{2}(y)=\frac{\alpha_{2}^{\theta_{2}}}{1-\alpha_{2}^{\theta_{2}}}\left[\sum_{k=0}^{\infty} \frac{\Gamma\left(\theta_{2}+\mathrm{k}\right)}{\Gamma\left(\theta_{2}\right) \mathrm{k} !}\left(1-\alpha_{2}\right)^{k} e^{-\lambda^{\beta} k y^{-\beta}}-1\right]
\end{gathered}
$$

We have

$$
\begin{aligned}
& \mathrm{R}=\int_{0}^{\infty} g_{1}(y) G_{2}(y) d y \\
& =\sum_{j=0}^{\infty} \sum_{k=0}^{\infty} \frac{\alpha_{1}^{\theta_{1}} \alpha_{2}^{\theta_{2}} \Gamma\left(\theta_{1}+\mathrm{j}+1\right) \Gamma\left(\theta_{2}+\mathrm{k}\right)\left(1-\alpha_{1}\right)^{j+1}\left(1-\alpha_{2}\right)^{k} \theta_{1} \beta \lambda^{\beta}}{\left(1-\alpha_{1}^{\theta_{1}}\right)\left(1-\alpha_{2}^{\theta_{2}}\right) \Gamma\left(\theta_{1}+1\right) \Gamma\left(\theta_{2}\right) \mathrm{j} ! \mathrm{k} !} \\
& \int_{0}^{\infty} y^{-(\beta+1)} e^{-\lambda^{\beta}(j+k) y^{-\beta}} d y-\frac{\alpha_{2}^{\theta_{2}}}{1-\alpha_{2}^{\theta_{2}}} \\
& =\sum_{j=0}^{\infty} \sum_{k=0}^{\infty} \frac{\alpha_{1}^{\theta_{1}} \alpha_{2}^{\theta_{2}} \Gamma\left(\theta_{1}+\mathrm{j}+1\right) \Gamma\left(\theta_{2}+\mathrm{k}\right)\left(1-\alpha_{1}\right)^{j+1}\left(1-\alpha_{2}\right)^{k} \theta_{1}}{\left(1-\alpha^{\theta_{1}}\right)\left(1-\alpha^{\theta_{2}}\right) \Gamma\left(\theta_{1}+1\right) \Gamma\left(\theta_{2}\right) \mathrm{j} ! \mathrm{k} !(\mathrm{j}+\mathrm{k}+1)}-\frac{\alpha_{2}^{\theta_{2}}}{1-\alpha_{2}^{\theta_{2}}} .
\end{aligned}
$$

Let us assume that $\mathrm{y}_{1}, \mathrm{y}_{2}, \ldots, \mathrm{y}_{\mathrm{n}}$ and $\mathrm{z}_{1}, \mathrm{z}_{2}, \ldots, \mathrm{z}_{\mathrm{m}}$ are independent observations from $\mathrm{Y}$ and $\mathrm{Z}$ respectively. The total $\log$-likelihood function $\mathrm{L}_{\mathrm{R}}\left(\Theta^{*}\right)$ where $\Theta^{*}=\left(\alpha_{1}, \alpha_{2}, \theta_{1}, \theta_{2}, \lambda, \beta\right)^{\mathrm{T}}$, becomes 


$$
\begin{aligned}
\mathrm{L}_{\mathrm{R}}\left(\Theta^{*}\right)=n \theta_{1} & \log \left(\alpha_{1}\right)+m \theta_{2} \log \left(\alpha_{2}\right)-n \log \left(1-\alpha_{1}^{\theta_{1}}\right)-m \log \left(1-\alpha_{2}^{\theta_{2}}\right)+n \log \left(\theta_{1}\right) \\
& +m \log \left(\theta_{2}\right)+(n+m) \log \left(\beta \lambda^{\beta}\right)-(\beta+1)\left[\sum_{i=1}^{n} \log \left(y_{i}\right)+\sum_{j=1}^{m} \log \left(z_{i}\right)\right] \\
& -\sum_{i=1}^{n}\left(\frac{\lambda}{\mathrm{y}_{\mathrm{i}}}\right)^{\beta}-\sum_{j=1}^{m}\left(\frac{\lambda}{\mathrm{z}_{\mathrm{j}}}\right)^{\beta}-\left(\theta_{1}+1\right) \sum_{i=1}^{n} \log \left[1-\left(1-\alpha_{1}\right) e^{\left.-\left(\frac{\lambda}{y_{i}}\right)^{\beta}\right]}\right. \\
& -\left(\theta_{2}+1\right) \sum_{j=1}^{m} \log \left[1-\left(1-\alpha_{2}\right) e^{\left.-\left(\frac{\lambda}{z_{j}}\right)^{\beta}\right] .}\right.
\end{aligned}
$$

By taking the first partial derivatives of the total log-likelihood function with respect to six parameters in $\Theta^{*}$, we obtain the six normal equations. The maximum likelihood estimates $\widehat{\Theta}^{*}$ of $\Theta^{*}$ is obtained by solving the system of non linear normal equations numerically. From the solution of these equations, we can estimate $\mathrm{R}$ by inserting the estimate of $\widehat{\Theta}^{*}$ in equation (34).

\section{Estimation of the parameters}

\subsection{Maximum likelihood estimation}

Several approaches for parameter estimation have been proposed in the literature, but maximum likelihood method is the most commonly employed. We consider estimation of the unknown parameters of GIW distribution by the method of maximum likelihood. Let $\mathrm{y}_{1}, \mathrm{y}_{2}, \ldots, \mathrm{y}_{\mathrm{n}}$ be observed values from the GIW distribution with parameters $\alpha, \theta, \lambda$ and $\beta$. The log-likelihood function for $(\alpha, \theta, \lambda, \beta)$ is given by

$$
\begin{gathered}
\log \mathrm{L}=n \log \left[\frac{\alpha^{\theta}(1-\alpha) \theta \beta \lambda^{\beta}}{1-\alpha^{\theta}}\right]-(\beta+1) \sum_{i=1}^{n} \log \left(y_{i}\right)-\sum_{i=1}^{n}\left(\frac{\lambda}{y_{\mathrm{i}}}\right)^{\beta} \\
-(\theta+1) \sum_{i=1}^{n} \log \left[1-(1-\alpha) e^{-\left(\frac{\lambda}{y_{i}}\right)^{\beta}}\right]
\end{gathered}
$$

The derivatives of the log-likelihood function with respect to the parameters $\alpha, \theta, \lambda$ and $\beta$ are given by respectively,

$$
\begin{gathered}
\frac{\partial \log \mathrm{L}}{\partial \alpha}=\frac{n \theta}{\alpha}-\frac{n}{1-\alpha}+\frac{n \theta \alpha^{\theta-1}}{1-\alpha^{\theta}}-\sum_{i=1}^{n} \frac{(\theta+1) e^{-\left(\frac{\lambda}{y_{i}}\right)^{\beta}}}{1-(1-\alpha) e^{-\left(\frac{\lambda}{y_{i}}\right)^{\beta}}} \\
\frac{\partial \log \mathrm{L}}{\partial \theta}=n \log (\alpha)-\frac{n}{\theta}+\frac{n \alpha^{\theta} \log (\alpha)}{1-\alpha^{\theta}}-\sum_{i=1}^{n} \log \left[1-(1-\alpha) e^{-\left(\frac{\lambda}{y_{i}}\right)^{\beta}}\right]
\end{gathered}
$$




$$
\begin{gathered}
\frac{\partial \log \mathrm{L}}{\partial \lambda}=\frac{n \beta}{\lambda}-\frac{\beta}{\lambda} \sum_{i=1}^{n}\left(\frac{\lambda}{\mathrm{y}_{\mathrm{i}}}\right)^{\beta}-\sum_{i=1}^{n} \frac{(\theta+1)(1-\alpha) \frac{\beta}{\lambda}\left(\frac{\lambda}{\mathrm{y}_{\mathrm{i}}}\right)^{\beta} e^{-\left(\frac{\lambda}{y_{i}}\right)^{\beta}}}{1-(1-\alpha) e^{-\left(\frac{\lambda}{y_{i}}\right)^{\beta}}} \\
\frac{\partial \log \mathrm{L}}{\partial \beta}=\frac{n}{\beta}+n \log (\lambda)-\sum_{i=1}^{n} \log \left(y_{i}\right)-\sum_{i=1}^{n}\left(\frac{\lambda}{\mathrm{y}_{\mathrm{i}}}\right)^{\beta} \log \left(\frac{\lambda}{\mathrm{y}_{\mathrm{i}}}\right) \\
-\sum_{i=1}^{n} \frac{(\theta+1)(1-\alpha)\left(\frac{\lambda}{\mathrm{y}_{\mathrm{i}}}\right)^{\beta} \log \left(\frac{\lambda}{\mathrm{y}_{\mathrm{i}}}\right) e^{-\left(\frac{\lambda}{y_{i}}\right)^{\beta}}}{1-(1-\alpha) e^{-\left(\frac{\lambda}{y_{i}}\right)^{\beta}}}
\end{gathered}
$$

The maximum likelihood estimates of $(\alpha, \theta, \lambda, \beta)$, say $(\widehat{\alpha}, \hat{\theta}, \hat{\lambda}, \widehat{\beta})$ are the simultaneous solutions of the equation $\frac{\partial \log \mathrm{L}}{\partial \alpha}=0, \frac{\partial \log \mathrm{L}}{\partial \theta}=0, \frac{\partial \log \mathrm{L}}{\partial \lambda}$, and $\frac{\partial \log \mathrm{L}}{\partial \beta}=0$. Maximization of the likelihood function can be performed by using $n l m$ or optim in R statistical package.

Now, we study the existence and uniqueness of the maximum likelihood estimates, when the other parameters are known (given).

Theorem 6.1: Let $g_{1}(\alpha ; \theta, \lambda, \beta, y)$ denote the function on the right-hand-side (RHS) of equation (35), where $\theta, \lambda, \beta$ are the true value of the parameters. Then there exist a solution for $g_{1}(\alpha ; \theta, \lambda, \beta, y)=0$ for $\alpha \in(0, \infty)$ and the solution is unique when $A_{1}>B_{1}$, where $A_{1}=\frac{n \theta}{\alpha^{2}}+\frac{n}{(1-\alpha)^{2}}+$ $\frac{n \theta \alpha^{\theta-1}\left(1-\theta-\alpha^{\theta}\right)}{\alpha\left(1-\alpha^{\theta}\right)^{2}}$ and $B_{1}=\sum_{i=1}^{n} \frac{(\theta+1)\left(e^{-\left(\frac{\lambda}{y_{i}}\right)^{\beta}}\right)^{2}}{\left[1-(1-\alpha) e^{-\left(\frac{\lambda}{y_{i}}\right)^{\beta}}\right]^{2}}$

Proof: We have

$$
g_{1}(\alpha ; \theta, \lambda, \beta, y)=\frac{n \theta}{\alpha}-\frac{n}{1-\alpha}+\frac{n \theta \alpha^{\theta-1}}{1-\alpha^{\theta}}-\sum_{i=1}^{n} \frac{(\theta+1) e^{-\left(\frac{\lambda}{y_{i}}\right)^{\beta}}}{1-(1-\alpha) e^{-\left(\frac{\lambda}{y_{i}}\right)^{\beta}}}
$$

Now

$$
\lim _{\alpha \rightarrow 0} g_{1}(\alpha ; \theta, \lambda, \beta, y)=\infty-n-0-\sum_{i=1}^{n} \frac{(\theta+1) e^{-\left(\frac{\lambda}{y_{i}}\right)^{\beta}}}{1-(1-\alpha) e^{-\left(\frac{\lambda}{y_{i}}\right)^{\beta}}}=\infty
$$

Also 


$$
\lim _{\alpha \rightarrow \infty} g_{1}(\alpha ; \theta, \lambda, \beta, y)=0-0-0-\lim _{\alpha \rightarrow \infty} \sum_{i=1}^{n} \frac{(\theta+1) e^{-\left(\frac{\lambda}{y_{i}}\right)^{\beta}}}{1-(1-\alpha) e^{-\left(\frac{\lambda}{y_{i}}\right)^{\beta}}}<0 .
$$

Therefore, there exist at least one root say $\widehat{\alpha} \in(0, \infty)$ such that $g_{1}(\widehat{\alpha} ; \theta, \lambda, \beta, y)=0$.

For uniqueness of root, the first derivative of $g_{1}(\alpha ; \theta, \lambda, \beta, y)$ is $\frac{\partial g_{1}(\alpha ; \theta, \lambda, \beta, y)}{\partial \alpha}$

$$
\begin{aligned}
& =-\frac{n \theta}{\alpha^{2}}-\frac{n}{(1-\alpha)^{2}}-\frac{n \theta \alpha^{\theta-1}\left(1-\theta-\alpha^{\theta}\right)}{\alpha\left(1-\alpha^{\theta}\right)^{2}}+\sum_{i=1}^{n} \frac{(\theta+1)\left(e^{-\left(\frac{\lambda}{y_{i}}\right)^{\beta}}\right)^{2}}{\left[1-(1-\alpha) e^{-\left(\frac{\lambda}{y_{i}}\right)^{\beta}}\right]^{2}} \\
& <0
\end{aligned}
$$

when $A_{1}>B_{1}$.

So there exist a solution for $g_{1}(\alpha ; \theta, \lambda, \beta, y)=0$ and the solution is unique when $A_{1}>B_{1}$.

Theorem 6.2: Let $g_{2}(\theta ; \alpha, \lambda, \beta, y)$ denote the function on the RHS of equation (36), where $\alpha, \lambda, \beta$ are the true value of the parameters. Then there exist a solution for $g_{2}(\theta ; \alpha, \lambda, \beta, y)=0$ for $\theta \in(0, \infty)$. The solution is unique when $\frac{n}{\theta^{2}}+\frac{n \alpha^{\theta} \log (\alpha)^{2}}{\left(1-\alpha^{\theta}\right)^{2}}<0$.

Proof: We have

$$
g_{2}(\theta ; \alpha, \lambda, \beta, y)=\frac{n \beta}{\lambda}-\frac{\beta}{\lambda} \sum_{i=1}^{n}\left(\frac{\lambda}{\mathrm{y}_{\mathrm{i}}}\right)^{\beta}-\sum_{i=1}^{n} \frac{(\theta+1)(1-\alpha) \frac{\beta}{\lambda}\left(\frac{\lambda}{\mathrm{y}_{\mathrm{i}}}\right)^{\beta} e^{-\left(\frac{\lambda}{y_{i}}\right)^{\beta}}}{1-(1-\alpha) e^{-\left(\frac{\lambda}{y_{i}}\right)^{\beta}}} .
$$

Then

$$
\lim _{\theta \rightarrow 0} g_{2}(\theta ; \alpha, \lambda, \beta, y)=n \log (\alpha)-\infty-\infty-\sum_{i=1}^{n} \log \left[1-(1-\alpha) e^{-\left(\frac{\lambda}{y_{i}}\right)^{\beta}}\right]=-\infty .
$$

On the other hand, when $\alpha<1$,

$$
\lim _{\theta \rightarrow \infty} g_{2}(\theta ; \alpha, \lambda, \beta, y)=n \log (\alpha)-0-0-\sum_{i=1}^{n} \log \left[1-(1-\alpha) e^{-\left(\frac{\lambda}{y_{i}}\right)^{\beta}}\right]>0 .
$$

Therefore, there exist at least one root say $\hat{\theta}$ such that $g_{2}(\theta ; \alpha, \lambda, \beta, y)=0$. 
Also

$$
\frac{\partial g_{2}(\theta ; \alpha, \lambda, \beta, y)}{\partial \theta}=\frac{\mathbf{n}}{\theta^{2}}+\frac{n \alpha^{\theta} \log (\alpha)^{2}}{\left(1-\alpha^{\theta}\right)^{2}}
$$

Hence the root is unique when equation $(39)<0$.

Theorem 6.3: Let $g_{3}(\lambda ; \alpha, \theta, \beta, y)$ denote the function on the RHS of equation (37), where $\alpha, \theta, \beta$ are the true value of the parameters. Then there exist a solution for $g_{3}(\lambda ; \alpha, \theta, \beta, y)=0$ for $\lambda \in(0, \infty)$. The solution is unique when $A_{3}<0$, where

$\mathrm{A}_{3}$

$=-\frac{n \beta}{\lambda^{2}}-\frac{\beta(\beta-1)}{\lambda^{2}} \sum_{i=1}^{n}\left(\frac{\lambda}{y_{i}}\right)^{\beta}$

$-\sum_{i=1}^{n} \frac{(\theta+1)(1-\alpha) \beta(\beta-1) \lambda^{2}\left(\frac{\lambda}{\mathrm{y}_{\mathrm{i}}}\right)^{\beta} e^{-\left(\frac{\lambda}{y_{i}}\right)^{\beta}\left[1-\beta\left(\frac{\lambda}{\mathrm{y}_{\mathrm{i}}}\right)^{\beta}-(1-\alpha) e^{-\left(\frac{\lambda}{y_{i}}\right)^{\beta}}\right]}}{\left[1-(1-\alpha) e^{\left.-\left(\frac{\lambda}{y_{i}}\right)^{\beta}\right]^{2}}\right.}$.

Proof: We have

$$
g_{3}(\lambda ; \alpha, \theta, \beta, y)=\frac{n \beta}{\lambda}-\frac{\beta}{\lambda} \sum_{i=1}^{n}\left(\frac{\lambda}{y_{\mathrm{i}}}\right)^{\beta}-\sum_{i=1}^{n} \frac{(\theta+1)(1-\alpha) \frac{\beta}{\lambda}\left(\frac{\lambda}{y_{\mathrm{i}}}\right)^{\beta} e^{-\left(\frac{\lambda}{y_{i}}\right)^{\beta}}}{1-(1-\alpha) e^{-\left(\frac{\lambda}{y_{i}}\right)^{\beta}}} .
$$

Now

$$
\lim _{\lambda \rightarrow 0} g_{3}(\lambda ; \alpha, \theta, \beta, y)=\infty-0-0=\infty
$$

and

$$
\lim _{\lambda \rightarrow \infty} g_{3}(\lambda ; \alpha, \theta, \beta, y)=0-\infty-\infty=-\infty
$$

Therefore, there exist at least one root say $\hat{\lambda} \in(0, \infty)$ such that $g_{3}(\hat{\lambda} ; \alpha, \theta, \beta, y)=0$. The root is unique when

$$
\frac{\partial g_{3}(\lambda ; \alpha, \theta, \beta, y)}{\partial \lambda}=-\frac{n \beta}{\lambda^{2}}-\frac{\beta(\beta-1)}{\lambda^{2}} \sum_{i=1}^{n}\left(\frac{\lambda}{y_{\mathrm{i}}}\right)^{\beta}-
$$




$$
\sum_{i=1}^{n} \frac{(\theta+1)(1-\alpha) \beta(\beta-1) \lambda^{2}\left(\frac{\lambda}{y_{\mathrm{i}}}\right)^{\beta} e^{-\left(\frac{\lambda}{y_{i}}\right)^{\beta}\left[1-\beta\left(\frac{\lambda}{\mathrm{y}_{\mathrm{i}}}\right)^{\beta}-(1-\alpha) e^{-\left(\frac{\lambda}{y_{i}}\right)^{\beta}}\right]}}{\left[1-(1-\alpha) e^{\left.-\left(\frac{\lambda}{y_{i}}\right)^{\beta}\right]^{2}}\right.}<0
$$

Theorem 6.4: Let $g_{4}(\beta ; \alpha, \theta, \lambda, y)$ denote the function on the RHS of equation (38), where $\alpha, \theta, \lambda$ are the true value of the parameters. Then there exist a solution for $g_{4}(\beta ; \alpha, \theta, \lambda, y)=0$ for $\beta \in(0, \infty)$. The solution is unique when $A_{4}<0$, where

$$
\begin{aligned}
& \mathrm{A}_{4}=-\frac{n}{\beta^{2}}-\sum_{i=1}^{n}\left(\frac{\lambda}{\mathrm{y}_{\mathrm{i}}}\right)^{\beta} \log \left(\frac{\lambda}{\mathrm{y}_{\mathrm{i}}}\right)^{2}- \\
& \sum_{i=1}^{n} \frac{(\theta+1)(1-\alpha)\left(\frac{\lambda}{y_{\mathrm{i}}}\right)^{\beta} \log \left(\frac{\lambda}{\mathrm{y}_{\mathrm{i}}}\right)^{2} e^{-\left(\frac{\lambda}{y_{i}}\right)^{\beta}\left[1-(1-\alpha) e^{-\left(\frac{\lambda}{y_{i}}\right)^{\beta}}-\left(\frac{\lambda}{\mathrm{y}_{\mathrm{i}}}\right)^{\beta}\right]}}{\left[1-(1-\alpha) e^{\left.-\left(\frac{\lambda}{y_{i}}\right)^{\beta}\right]^{2}}\right.}
\end{aligned}
$$

Proof: We have

$$
\begin{gathered}
g_{4}(\beta ; \alpha, \theta, \lambda, y)=\frac{n}{\beta}+n \log (\lambda)-\sum_{i=1}^{n} \log \left(y_{i}\right)-\sum_{i=1}^{n}\left(\frac{\lambda}{\mathrm{y}_{\mathrm{i}}}\right)^{\beta} \log \left(\frac{\lambda}{\mathrm{y}_{\mathrm{i}}}\right) \\
-\sum_{i=1}^{n} \frac{(\theta+1)(1-\alpha)\left(\frac{\lambda}{\mathrm{y}_{\mathrm{i}}}\right)^{\beta} \log \left(\frac{\lambda}{\mathrm{y}_{\mathrm{i}}}\right) e^{-\left(\frac{\lambda}{y_{i}}\right)^{\beta}}}{1-(1-\alpha) e^{-\left(\frac{\lambda}{y_{i}}\right)^{\beta}}} .
\end{gathered}
$$

Now

$$
\begin{gathered}
\lim _{\beta \rightarrow 0} g_{4}(\beta ; \alpha, \theta, \lambda, y)=\infty+n \log (\lambda)-\sum_{i=1}^{n} \log \left(y_{i}\right)-\sum_{i=1}^{n} \log \left(\frac{\lambda}{y_{\mathrm{i}}}\right) \\
-\sum_{i=1}^{n} \frac{(\theta+1)(1-\alpha) \log \left(\frac{\lambda}{y_{\mathrm{i}}}\right) e^{-1}}{1-(1-\alpha) e^{-1}}=\infty .
\end{gathered}
$$

On the other hand

$$
\lim _{\beta \rightarrow \infty} g_{4}(\beta ; \alpha, \theta, \lambda, y)=0+n \log (\lambda)-\sum_{i=1}^{n} \log \left(y_{i}\right)-\infty-0=-\infty .
$$


Therefore, there exist at least one root say $\hat{\beta} \in(0, \infty)$ such that $g_{4}(\widehat{\beta} ; \alpha, \theta, \lambda, y)=0$.

Also

$$
\begin{aligned}
& \frac{\partial g_{4}(\beta ; \alpha, \theta, \lambda, y)}{\partial \beta}=-\frac{n}{\beta^{2}}-\sum_{i=1}^{n}\left(\frac{\lambda}{y_{\mathrm{i}}}\right)^{\beta} \log \left(\frac{\lambda}{\mathrm{y}_{\mathrm{i}}}\right)^{2}- \\
& \sum_{i=1}^{n} \frac{(\theta+1)(1-\alpha)\left(\frac{\lambda}{\mathrm{y}_{\mathrm{i}}}\right)^{\beta} \log \left(\frac{\lambda}{\mathrm{y}_{\mathrm{i}}}\right)^{2} e^{-\left(\frac{\lambda}{y_{i}}\right)^{\beta}\left[1-(1-\alpha) e^{-\left(\frac{\lambda}{y_{i}}\right)^{\beta}}-\left(\frac{\lambda}{\mathrm{y}_{\mathrm{i}}}\right)^{\beta}\right]}}{\left[1-(1-\alpha) e^{\left.-\left(\frac{\lambda}{y_{i}}\right)^{\beta}\right]^{2}}\right.} .
\end{aligned}
$$

Hence there exist a unique solution for $g_{4}(\widehat{\beta} ; \alpha, \theta, \lambda, y)=0$, when equation (40) $<0$.

The normal approximation of the maximum likelihood estimates of the parameters can be adopted for constructing approximate confidence intervals and for testing hypotheses on the parameters $(\alpha, \theta, \lambda, \beta)$. Under conditions that are fulfilled for the parameters in the interior of the parameter space and applying the usual large sample approximation, it can be shown that $\sqrt{\mathrm{n}}(\Theta-\widehat{\Theta})$ can be approximated by a multivariate normal distribution with zero means and variance-covariance matrix $\mathrm{K}^{-1}(\Theta)$, where $\mathrm{K}(\Theta)$ is the unit expected information matrix.

As $\mathrm{n}$ tends to infinity, we have the asymptotic result

$$
\mathrm{K}(\Theta)=\lim _{n \rightarrow \infty} \frac{1}{n} I(\Theta)
$$

where $I(\Theta)$ is the observed Fisher information matrix. Since $K(\Theta)$ involves the unknown parameters of $\Theta$, we may replace it with the MLE $\widehat{\Theta}$. Thus, the average matrix estimated at $\widehat{\Theta}$, say $\frac{1}{n} I(\Theta)$, can be used to estimate $K(\Theta)$. The estimated multivariate normal distribution can thus be used to construct approximate confidence intervals for the unknown parameters and for the hazard rate and survival function.

\subsection{Method of product spacing}

This method was introduced by Cheng and Amin (1983) as an alternative to method of maximum likelihood estimation. The cdf of the GIW distribution is given by equation (18), and the uniform spacing are defined as follows: 


$$
\begin{gathered}
\mathrm{D}_{1}=\mathrm{G}\left(\mathrm{y}_{1}\right)=\frac{\alpha^{\theta}}{1-\alpha^{\theta}}\left[\left[1-(1-\alpha) e^{\left.\left.-\left(\frac{\lambda}{y_{1}}\right)^{\beta}\right]^{-\theta}-1\right]}\right.\right. \\
\mathrm{D}_{\mathrm{n}+1}=1-\mathrm{G}\left(\mathrm{y}_{\mathrm{n}}\right)=1-\frac{\alpha^{\theta}}{1-\alpha^{\theta}}\left[\left[1-(1-\alpha) e^{-\left(\frac{\lambda}{y_{n}}\right)^{\beta}}\right]^{-\theta}-1\right],
\end{gathered}
$$

and the general term of spacing is given by

$$
\begin{aligned}
\mathrm{D}_{\mathrm{i}}=\mathrm{G}\left(\mathrm{y}_{\mathrm{i}}\right) & -\mathrm{G}\left(\mathrm{y}_{\mathrm{i}-1}\right)=\frac{\alpha^{\theta}}{1-\alpha^{\theta}}\left[\left[1-(1-\alpha) e^{-\left(\frac{\lambda}{y_{i}}\right)^{\beta}}\right]^{-\theta}\right. \\
& \left.-\left[1-(1-\alpha) e^{-\left(\frac{\lambda}{y_{i-1}}\right)^{\beta}}\right]^{-\theta}-1\right]
\end{aligned}
$$

such that $\sum D_{i}=1$. Method of product spacing method choose the estimates which maximizes the product of spacings or in other words, maximizes the geometric mean of the spacing. That is,

$$
\mathrm{G}=\left(\prod_{i=1}^{n+1} D_{i}\right)^{\frac{1}{\mathrm{n}+1}}
$$

Taking the logarithm of $\mathrm{G}$, we get,

$$
S=\frac{1}{n+1} \sum_{i=1}^{n+1} \log \left(D_{i}\right), \text { where } S=\log G
$$

We can rewrite $\mathrm{S}$ as

$$
\begin{gathered}
\mathrm{S}=\frac{1}{\mathrm{n}+1}\left\{\log \left(D_{1}\right)+\sum_{i=2}^{n} \log \left(D_{i}\right)+\log \left(D_{n+1}\right)\right\} \\
=\frac{1}{\mathrm{n}+1} \log \left(\frac { \alpha ^ { \theta } } { 1 - \alpha ^ { \theta } } \left\{\left[1-(1-\alpha) e^{\left.\left.\left.-\left(\frac{\lambda}{y_{1}}\right)^{\beta}\right]^{-\theta}-1\right\}\right)+}\right.\right.\right. \\
\frac{1}{\mathrm{n}+1} \sum_{\mathrm{i}=2}^{\mathrm{n}} \log \left(\frac { \alpha ^ { \theta } } { 1 - \alpha ^ { \theta } } \left\{\left[1-(1-\alpha) e^{\left.\left.-\left(\frac{\lambda}{y_{i}}\right)^{\beta}\right]^{-\theta}-\left[1-(1-\alpha) e^{\left.-\left(\frac{\lambda}{y_{i-1}}\right)^{\beta}\right]^{-\theta}}\right\}\right)}\right.\right.\right. \\
+\frac{1}{\mathrm{n}+1} \log \left(\frac{1-\alpha^{\theta}\left[1-(1-\alpha) e^{\left.-\left(\frac{\lambda}{y_{n}}\right)^{\beta}\right]^{-\theta}}\right.}{1-\alpha^{\theta}}\right) .
\end{gathered}
$$

Differentiating the above equation partially, with respect to the parameters $\alpha, \theta, \lambda$ and $\beta$ respectively and then equating them to zero, we get the normal equations. 
Since the normal equations are non-linear, we can use iterative method to obtain the solution.

\subsection{Method of least squares}

Let $\mathrm{y}_{1}<\mathrm{y}_{2}<\cdots<\mathrm{y}_{\mathrm{n}}$ be the $\mathrm{n}$ ordered random sample of any distribution with cdf $\mathrm{G}(\mathrm{y})$, we get

$$
E\left[G\left(y_{i}\right)\right]=\frac{i}{n+1}
$$

The least square estimates are obtained by minimizing

$$
P(\alpha, \theta, \lambda, \beta)=\sum_{i=1}^{n}\left(G\left(y_{i}\right)-\frac{i}{n+1}\right)^{2} .
$$

Putting the cdf of GIW distribution in equation (41), we get

$$
\mathrm{P}(\alpha, \theta, \lambda, \beta)=\sum_{\mathrm{i}=1}^{\mathrm{n}}\left(\frac{\alpha^{\theta}}{1-\alpha^{\theta}}\left\{\frac{1-\left[1-(1-\alpha) e^{-\left(\frac{\lambda}{y_{i}}\right)^{\beta}}\right]^{\theta}}{\left[1-(1-\alpha) e^{\left.-\left(\frac{\lambda}{y_{i}}\right)^{\beta}\right]^{\theta}}\right.}\right\}-\frac{\mathrm{i}}{\mathrm{n}+1}\right)^{2}
$$

In order to minimize equation (42), we have to differentiate it with respect to $\alpha, \theta, \lambda$ and $\beta$, which gives the following equations:

$$
\begin{aligned}
& \sum_{i=1}^{n} \frac{\alpha^{\theta}}{1-\alpha^{\theta}}\left(\frac{1-A^{\theta}}{A^{\theta}}-\frac{i}{n+1}\right)\left(\frac{\alpha^{\theta} \theta\left(1-A^{\theta}\right)}{\alpha A^{\theta}\left(1-\alpha^{\theta}\right)}\left[1+\frac{\alpha^{\theta}}{1-\alpha^{\theta}}\right]\right. \\
& \left.-\frac{\alpha^{\theta} \theta e^{-\left(\frac{\lambda}{y_{i}}\right)^{\beta}}}{\mathrm{A}\left(1-\alpha^{\theta}\right)}\left[1+\frac{1-\mathrm{A}^{\theta}}{\mathrm{A}^{\theta}}\right]\right)=0 \\
& \sum_{i=1}^{n} \frac{\alpha^{\theta}}{1-\alpha^{\theta}}\left(\frac{1-A^{\theta}}{A^{\theta}}-\frac{i}{n+1}\right)\left(\frac{\alpha^{\theta}\left(1-A^{\theta}\right) \log (\alpha)}{A^{\theta}\left(1-\alpha^{\theta}\right)}\left[1+\frac{\alpha^{\theta}}{1-\alpha^{\theta}}\right]\right. \\
& \left.-\frac{\alpha^{\theta} \log (A)}{1-\alpha^{\theta}}\left[1+\frac{1-\mathrm{A}^{\theta}}{\mathrm{A}^{\theta}}\right]\right)=0 \\
& \sum_{i=1}^{n} \frac{\alpha^{\theta}}{1-\alpha^{\theta}}\left(\frac{1-A^{\theta}}{A^{\theta}}-\frac{i}{n+1}\right)\left(\frac{\alpha^{\theta} \theta(1-\alpha)\left(\frac{\beta}{\lambda}\right)\left(\frac{\lambda}{y_{i}}\right)^{\beta} e^{-\left(\frac{\lambda}{y_{i}}\right)^{\beta}}}{A\left(1-\alpha^{\theta}\right)}\left[1+\frac{1-\mathrm{A}^{\theta}}{\mathrm{A}^{\theta}}\right]\right)=0
\end{aligned}
$$


$\sum_{i=1}^{n} \frac{\alpha^{\theta}}{1-\alpha^{\theta}}\left(\frac{1-A^{\theta}}{A^{\theta}}-\frac{i}{n+1}\right)\left(\frac{\alpha^{\theta} \theta(1-\alpha)\left(\frac{\lambda}{y_{i}}\right)^{\beta} \log \left(\frac{\lambda}{y_{i}}\right) e^{-\left(\frac{\lambda}{y_{i}}\right)^{\beta}}}{A\left(1-\alpha^{\theta}\right)}\left[1+\frac{1-\mathrm{A}^{\theta}}{\mathrm{A}^{\theta}}\right]\right)=0$

where $\mathrm{A}=1-(1-\alpha) e^{-\left(\frac{\lambda}{y_{i}}\right)^{\beta}}$. The above normal equations cannot be solved analytically. So we can use $n l m$ or optim in R statistical package to obtain the solution.

\subsection{Simulation}

We asses the performance of the maximum likelihood estimates of GIW $(\alpha, \theta, \lambda, \beta)$ distribution by conducting simulation for different sample sizes and parameter values. We use equation (29) to generate random samples from the GIW distribution with parameters $\alpha, \theta, \lambda$ and $\beta$. The different sample sizes considered in the simulation are $n=30,70,100$ and 200 . We have used nlm package in $\mathrm{R}$ software to find the estimate. We have repeated the process 1000 times and report the average estimates and associated mean square errors in Table 3.

Table 3: Simulation results for different values of the parameters $\alpha, \theta, \lambda$ and $\beta$.

\begin{tabular}{|c|l|l|l|l|l|}
\hline$n$ & & $\widehat{\alpha}(\operatorname{MSE}(\widehat{\alpha}))$ & $\hat{\theta}(\operatorname{MSE}(\hat{\theta}))$ & $\widehat{\lambda}(\operatorname{MSE}(\hat{\lambda}))$ & $\hat{\beta}(\operatorname{MSE}(\widehat{\beta}))$ \\
& & & & & \\
\hline & & & & & \\
70 & $\alpha=0.5$ & $0.599(0.295)$ & $0.573(1.056)$ & $1.234(0.267)$ & $1.857(0.028)$ \\
70 & $\theta=0.5$ & $0.483(0.028)$ & $0.580(0.841)$ & $1.056(0.145)$ & $1.452(0.015)$ \\
100 & $\lambda=1.0$ & $0.563(0.016)$ & $0.432(0.184)$ & $0.984(0.127)$ & $1.564(0.011)$ \\
200 & $\beta=1.5$ & $0.541(0.012)$ & $0.506(0.071)$ & $1.051(0.112)$ & $1.487(0.009)$ \\
& & & & & \\
\hline & & & & & \\
70 & $\alpha=1.5$ & $1.539(0.295)$ & $0.487(0.267)$ & $1.341(0.243)$ & $1.314(2.429)$ \\
100 & $\lambda=0.5$ & $1.578(0.244)$ & $0.522(0.165)$ & $0.896(1.420)$ & $2.607(1.161)$ \\
200 & $\beta=1.0$ & $1.560(0.196)$ & $0.476(0.093)$ & $1.254(0.937)$ & $1.503(1.026)$ \\
& & $1.553(0.144)$ & $0.523(0.051)$ & $1.187(0.532)$ & $2.224(0.764)$ \\
\hline
\end{tabular}




\begin{tabular}{|c|l|l|l|l|l|}
\hline 30 & $\alpha=0.5$ & $0.829(4.329)$ & $1.554(0.507)$ & $0.831(2.354)$ & $1.451(0.106)$ \\
70 & $\theta=1.5$ & $0.432(3.012)$ & $1.468(0.302)$ & $0.776(1.423)$ & $1.536(0.084)$ \\
100 & $\lambda=1.0$ & $0.306(2.019)$ & $1.547(0.187)$ & $0.813(0.937)$ & $1.458(0.065)$ \\
200 & $\beta=1.5$ & $0.458(1.282)$ & $1.459(0.103)$ & $0.913(0.532)$ & $1.567(0.042)$ \\
\hline & & & & & \\
30 & $\alpha=5.0$ & $6.265(5.661)$ & $1.732(0.159)$ & $1.541(1.056)$ & $2.191(0.645)$ \\
70 & $\theta=1.5$ & $4.847(5.062)$ & $1.624(0.127)$ & $1.320(0.841)$ & $1.936(0.391)$ \\
100 & $\lambda=1.0$ & $0.304(0.109)$ & $1.485(0.098)$ & $1.023(0.821)$ & $2.264(0.169)$ \\
200 & $\beta=2.0$ & $0.085(0.021)$ & $1.526(0.074)$ & $0.994(0.563)$ & $2.185(0.114)$ \\
\hline
\end{tabular}

From Table 3, we can see that as the sample size increase, the estimated values are close to the actual values and the mean square errors decreases, which establishes the consistency property of the MLEs.

\section{Autoregressive GIW minification process}

We develop a first order autoregressive $(\operatorname{AR}(1))$ minification process with GIW distribution as marginal distribution.

Consider an $\mathrm{AR}(1)$ minification process with structure

$$
\mathrm{Y}_{\mathrm{n}}=\left\{\begin{array}{c}
\epsilon_{n} \quad w \cdot p \rho \\
\min \left(Y_{n-1}, \epsilon_{n}\right) \quad w \cdot p 1-\rho
\end{array}\right.
$$

where $0<\rho<1, \mathrm{n} \geq 1$ and $\left\{\epsilon_{\mathrm{n}}\right\}$ is a sequence of i.i.d. random variables.

In order to develop time series models with GIW marginals, we need the following definition.

Definition 7.1: A random variable $X$ on $(0, \infty)$ is said to have Marshall-Olkin inverse truncated negative binomial Weibull (MOGIW) distribution and write as $X={ }^{d} \operatorname{MOGIW}(\nu, \alpha, \theta, \lambda, \beta)$ if it has the survival function

$$
\overline{\mathrm{F}}(x)=\frac{1}{1+\frac{1}{v}\left(\frac{\left[1-(1-\alpha) e^{\left.-\left(\frac{\lambda}{y}\right)^{\beta}\right]^{\theta}}-\alpha^{\theta}\right.}{\left(1-\alpha^{\theta}\right)\left[1-(1-\alpha) e^{\left.-\left(\frac{\lambda}{y}\right)^{\beta}\right]^{\theta}}\right.}\right)}
$$

Theorem 7.1: The AR(1) process given by (43) defines a stationary AR(1) minification process with $\operatorname{GIW}(\alpha, \theta, \lambda, \beta)$ as marginal distribution if and only if 
$\epsilon_{n}^{\prime}$ s are i.i.d. $\operatorname{MOGIW}\left(\rho^{-1}, \alpha, \theta, \lambda, \beta\right)$ with $Y_{0}={ }^{d} \operatorname{GIW}(\alpha, \theta, \lambda, \beta)$.

Proof: We have, for $G I W(\alpha, \theta, \lambda, \beta)$,

$$
\begin{gathered}
\overline{\mathrm{G}}_{Y}(y)=\frac{\left[1-(1-\alpha) e^{\left.-\left(\frac{\lambda}{y}\right)^{\beta}\right]^{\theta}}-\alpha^{\theta}\right.}{\left(1-\alpha^{\theta}\right)\left[1-(1-\alpha) e^{\left.-\left(\frac{\lambda}{y}\right)^{\beta}\right]^{\theta}}\right.} \\
=\frac{\alpha^{\theta}\left\{1-\left[1-(1-\alpha) e^{\left.\left.-\left(\frac{\lambda}{y}\right)^{\beta}\right]^{\theta}\right\}}\right.\right.}{\left[1-(1-\alpha) e^{\left.-\left(\frac{\lambda}{y}\right)^{\beta}\right]^{\theta}-\alpha^{\theta}}\right.}
\end{gathered}
$$

The model (43) can be rewritten in terms of survival function as

$$
\mathrm{P}\left(\mathrm{Y}_{\mathrm{n}}>y\right)=\mathrm{P}\left(\epsilon_{n}>y\right)\left[\rho+(1-\rho) \mathrm{P}\left(\mathrm{Y}_{\mathrm{n}-1}>y\right)\right] .
$$

That is,

$$
\overline{\mathrm{G}}_{Y_{n}}(y)=\overline{\mathrm{G}}_{\epsilon_{n}}(y)\left[\rho+(1-\rho) \overline{\mathrm{G}}_{Y_{n-1}}(y)\right]
$$

If $\left\{Y_{n}\right\}$ is stationary with $\operatorname{GIW}(\alpha, \theta, \lambda, \beta)$ marginals, then

$$
\overline{\mathrm{G}}_{\epsilon_{n}}(y)=\frac{\overline{\mathrm{G}}_{Y}(y)}{\rho+(1-\rho) \overline{\mathrm{G}}_{Y}(y)}=\frac{\frac{\left[1-(1-\alpha) e^{\left.-\left(\frac{\lambda}{y}\right)^{\beta}\right]^{\theta}-\alpha^{\theta}}\right.}{\left(1-\alpha^{\theta}\right)\left[1-(1-\alpha) e^{\left.-\left(\frac{\lambda}{y}\right)^{\beta}\right]^{\theta}}\right.}}{=\frac{\left[1-(1-\alpha) e^{\left.-\left(\frac{\lambda}{y}\right)^{\beta}\right]^{\theta}}-\alpha^{\theta}\right.}{\alpha^{\theta}\left\{1-\left[1-(1-\alpha) \frac{\left.\left(1-\alpha^{\theta}\right)\left[1-(1-\alpha) e^{-\left(\frac{\lambda}{y}\right)^{\beta}}\right]^{-\left(\frac{\lambda}{y}\right)^{\beta}}\right]^{\theta}}{\left[1-(1-\alpha) e^{-\left(\frac{\lambda}{y}\right)^{\beta}}\right]^{\theta}-\alpha^{\theta}}\right.\right.}}
$$


That is, $\epsilon^{\prime}{ }_{n}$ s are i.i.d. $\operatorname{MOGIW}\left(\rho^{-1}, \alpha, \theta, \lambda, \beta\right)$.

Conversely, if $\epsilon_{n}^{\prime}$ are i.i.d. $\operatorname{MOGIW}\left(\rho^{-1}, \alpha, \theta, \lambda, \beta\right)$ with $Y_{0}={ }^{d} \operatorname{GIW}(\alpha, \theta, \lambda, \beta)$, then from (44), we have

$$
\begin{aligned}
& \overline{\mathrm{G}}_{Y_{1}}(y)=\rho \overline{\mathrm{G}}_{\epsilon_{1}}(y)+(1-\rho) \overline{\mathrm{G}}_{\epsilon_{1}}(y) \overline{\mathrm{G}}_{Y_{0}}(y) \\
& 1+\rho \alpha^{\theta} \frac{\rho}{\left\{1-\left[1-(1-\alpha) e^{\left.\left.-\left(\frac{\lambda}{y}\right)^{\beta}\right]^{\theta}\right\}}\right.\right.}+(1-\rho) \\
& {\left[1-(1-\alpha) e^{\left.-\left(\frac{\lambda}{y}\right)^{\beta}\right]^{\theta}}-\alpha^{\theta}\right.}
\end{aligned}
$$

1

$1+\rho \alpha^{\theta} \frac{\left\{1-\left[1-(1-\alpha) e^{\left.\left.-\left(\frac{\lambda}{y}\right)^{\beta}\right]^{\theta}\right\}}\right.\right.}{\left[1-(1-\alpha) e^{\left.-\left(\frac{\lambda}{y}\right)^{\beta}\right]^{\theta}-\alpha^{\theta}}\right.} 1+\alpha^{\theta} \frac{\left\{1-\left[1-(1-\alpha) e^{\left.\left.-\left(\frac{\lambda}{y}\right)^{\beta}\right]^{\theta}\right\}}\right.\right.}{\left[1-(1-\alpha) e^{\left.-\left(\frac{\lambda}{y}\right)^{\beta}\right]^{\theta}-\alpha^{\theta}}\right.}$

$$
=\frac{\alpha^{\theta}\left\{1-\left[1-(1-\alpha) e^{\left.-\left(\frac{\lambda}{y}\right)^{\beta}\right]^{\theta}}\right\}\right.}{\left[1-(1-\alpha) e^{\left.-\left(\frac{\lambda}{y}\right)^{\beta}\right]^{\theta}}-\alpha^{\theta}\right.}
$$

$$
=\frac{\left[1-(1-\alpha) e^{-\left(\frac{\lambda}{y}\right)^{\beta}}\right]^{\theta}-\alpha^{\theta}}{\left(1-\alpha^{\theta}\right)\left[1-(1-\alpha) e^{-\left(\frac{\lambda}{y}\right)^{\beta}}\right]^{\theta}}
$$

That is, $Y_{1}={ }^{d} \operatorname{GIW}(\alpha, \theta, \lambda, \beta)$.

If we assume that $Y_{n-1}={ }^{d} \operatorname{GIW}(\alpha, \theta, \lambda, \beta)$, then by induction, we can establish that $Y_{n}={ }^{d} \operatorname{GIW}(\alpha, \theta, \lambda, \beta)$.

Hence the process $\left\{Y_{n}\right\}$ is stationary with GIW marginals.

\section{Application to real data}

In this section, we analyze one data set to demonstrate how the GIW distribution can be a good life time model in comparison with many known 
distributions. We consider the data set originally reported by Bjerkedal(1960). This data set consists of 72 observations of survival times guinea pigs injected with different doses of tubercle bacilli. The data set has been considered by several authors in the literature, see, Kundu and Howlader (2010) and Cordeiro et al. (2012). The data set follows:

1215222424323233343838434448525354545556575858596060 6060616263656567687070727375767681838485879195969899 109110121127129131143146146175175211233258258263297341341 376

The descriptive statistics of the data is presented in Table 4.

Table 4: Descriptive statistics of pig data.

\begin{tabular}{rcccccc}
\hline Min & Median & Mean & Max & SD & Skewness & Kurtosis \\
\hline 12.000 & 70.000 & 99.820 & 376.000 & 81.118 & 1.796 & 5.614 \\
\hline
\end{tabular}

The distribution of the data is positively skewed and leptokurtic. We compare the GIW distribution with the following life time distributions:

1. Inverse exponential (IE) distribution having the pdf

$$
g(\mathrm{y} ; \lambda)=\lambda \mathrm{y}^{-2} e^{-\frac{\lambda}{y}} ; \lambda>0, y>0 .
$$

2. Inverse Rayleigh (IR) distribution having the pdf

$$
g(\mathrm{y} ; \lambda)=2 \lambda^{2} \mathrm{y}^{-3} e^{-\left(\frac{\lambda}{y}\right)^{2}} ; \lambda>0, y>0 .
$$

3. Weibull (W) distribution having the pdf

$$
g(y ; \lambda, \beta)=\lambda^{\beta} \beta y^{\beta-1} e^{-(\lambda y)^{\beta}} ; \lambda, \beta>0, y>0 .
$$

4. Inverse Weibull (IW) distribution having the pdf

$$
g(y ; \lambda, \beta)=\lambda^{\beta} \beta y^{-(\beta+1)} e^{-\left(\frac{\lambda}{y}\right)^{\beta}} ; \lambda, \beta>0, y>0 .
$$

5. Exponentiated inverse Weibull (EIW) distribution having the pdf

$$
g(\mathrm{y} ; \alpha, \lambda, \beta)=\alpha \lambda^{\beta} \beta^{\alpha-1} \mathrm{y}^{-(\beta+1)} e^{-\left(\frac{\lambda}{y}\right)^{\beta}}\left[1-e^{-\left(\frac{\lambda}{y}\right)^{\beta}}\right]^{\alpha-1} ; \alpha, \lambda, \beta>0, y>0 .
$$

6. Inverse Weibull logarithmic (IWL) distribution having the pdf

$$
g(\mathrm{y} ; \alpha, \lambda, \beta)=\frac{\alpha \lambda \beta \mathrm{y}^{-(\beta+1)} e^{-\lambda y^{-\beta}}}{\left(1-\alpha e^{-\lambda y^{-\beta}}\right) \log (1-\alpha)^{-1}} ; 0<\alpha<1, \lambda, \beta>0, y>0 .
$$

7. Inverse Weibull Poisson (IWP) distribution having the pdf

$$
g(\mathrm{y} ; \alpha, \lambda, \beta)=\frac{e^{\alpha e^{-\lambda y^{-\beta}}}}{e^{\alpha}-1} \alpha \lambda \beta \mathrm{y}^{-(\beta+1)} e^{-\lambda y^{-\beta}} ;-\infty<\alpha<\infty, \lambda, \beta>0, y>0 .
$$


8. Inverse Marshall-Olkin Weibull (IMOW) distribution having the pdf

$$
g(\mathrm{y} ; \alpha, \lambda, \beta)=\frac{\alpha \lambda^{\beta} \beta \mathrm{y}^{-(\beta+1)} e^{-\left(\frac{\lambda}{y}\right)^{\beta}}}{\left[1-(1-\alpha) e^{-\left(\frac{\lambda}{y}\right)^{\beta}}\right]^{2}} ; \alpha, \lambda, \beta>0, y>0
$$

9. Kumaraswamy Marshall-Olkin inverse exponential (Kw-MOIE) distribution having the pdf

$$
\begin{gathered}
g(\mathrm{y} ; \mathrm{a}, \mathrm{b}, \alpha, \lambda)=a b \alpha \lambda x^{-2} e^{-a\left(\frac{\lambda}{y}\right)}\left[\alpha+(1-\alpha) e^{-\left(\frac{\lambda}{y}\right)}\right]^{-a-1} \\
{\left[1-e^{-a\left(\frac{\lambda}{y}\right)}\left[\alpha+(1-\alpha) e^{-\left(\frac{\lambda}{y}\right)}\right]^{-a}\right]^{b-1} ; a, b, \alpha, \lambda>0, y>0 .}
\end{gathered}
$$

10. Kumaraswamy Fréchet (Kw-Fr) distribution having the pdf

$$
\begin{aligned}
g(y ; a, b, \lambda, \beta) & =a b \beta \lambda^{\beta} y^{-\beta-1} e^{-a\left(\frac{\lambda}{y}\right)^{\beta}}\left[1-e^{-a\left(\frac{\lambda}{y}\right)^{\beta}}\right]^{b-1} ; a, b, \lambda, \beta>0, y \\
> & 0 .
\end{aligned}
$$

11. Beta Fréchet (BFr) distribution having the pdf

$$
\begin{aligned}
g(\mathrm{y} ; \mathrm{a}, \mathrm{b}, \lambda, \beta) & =\frac{\Gamma(a) \Gamma(b) \beta \lambda^{\beta}}{\Gamma(a+b)} y^{-\beta-1} e^{-a\left(\frac{\lambda}{y}\right)^{\beta}}\left[1-e^{-\left(\frac{\lambda}{y}\right)^{\beta}}\right]^{b-1} ; a, b, \lambda, \beta \\
>0, y>0 . &
\end{aligned}
$$

12. Exponentiated Weibull-Poisson (EWP) distribution having the pdf

$$
\begin{gathered}
g(\mathrm{y} ; \alpha, \lambda, \beta, \theta)=\frac{\alpha \theta \beta \lambda^{\beta}}{e^{\theta}-1} y^{\beta-1} e^{-(\lambda y)^{\beta}} \\
\left(1-e^{-(\lambda y)^{\beta}}\right)^{\alpha-1} e^{\theta\left(1-e^{-(\lambda y)^{\beta}}\right)^{\alpha}} ; \alpha, \lambda, \beta, \theta>0, y>0 .
\end{gathered}
$$

The parameters is estimated numerically using maximum likelihood estimate method. The values of $\log$-likelihood $(-\log L)$, Akaike information criterion (AIC), consistent Akaike information criterion (CAIC), Bayesian information criterion (BIC) and Hannan-Quinn information criterion (HQCI) statistics for GIW and their sub-models are calculated. The better distribution corresponds to smaller - $\log$ L, AIC, CAIC, BIC and HQCI.

We apply the Crammer-von $\operatorname{Mises}\left(\mathrm{W}^{*}\right)$ and Anderson-Darling $\left(\mathrm{A}^{*}\right)$ statistic for formal goodness-of-fit to verify which distribution fits better to this data. In general, the smaller the values of the statistics $\mathrm{W}^{*}$ and $\mathrm{A}^{*}$, shows better the fit to the data. Let $G(x ; \theta)$ be the cdf, where the form of $G$ is known but $\theta$ (a 4-dimensional parameter vector, say) is unknown. We calculate the statistics $\mathrm{W}^{*}$ and $A^{*}$ as follows:(i) Compute $\psi_{i}=G\left(x_{i} ; \hat{\theta}\right)$, where the $x_{i}$ 's are in ascending 
order; (ii) Compute $x_{i}=\phi^{-1}\left(\psi_{\mathrm{i}}\right)$, where $\phi($.$) is the normal cdf and \phi^{-1}($. its inverse.; (iii) Compute $\mathrm{u}_{\mathrm{i}}=\phi\left\{\left(\mathrm{x}_{\mathrm{i}}-\overline{\mathrm{x}}\right) / \mathrm{s}_{\mathrm{x}}\right\}$, where $\overline{\mathrm{x}}=\mathrm{n}^{-1} \sum_{\mathrm{i}=1}^{\mathrm{n}} \mathrm{x}_{\mathrm{i}}$ and $\mathrm{s}_{\mathrm{x}}^{2}=(\mathrm{n}-1)^{-1} \sum_{\mathrm{i}=1}^{\mathrm{n}}\left(\mathrm{x}_{\mathrm{i}}-\overline{\mathrm{x}}\right)^{2} ;$ (iv) Calculate

$$
W^{2}=\sum_{i=1}^{n}\left\{u_{i}-\frac{2 i-1}{2 n}\right\}^{2}+\frac{1}{12 n}
$$

and

$$
A^{2}=-n-\frac{1}{n} \sum_{i=1}^{n}\left\{(2 i-1) \log \left(u_{i}\right)+(2 n+1-2 i) \log \left(1-u_{i}\right)\right\} ;
$$

(v) Modify $W^{2}$ into $W^{*}=W^{2}(1+0.5 / n)$ and $A^{2}$ into $A^{*}=A^{2}(1+$ $\left.0.75 / \mathrm{n}+2.25 / \mathrm{n}^{2}\right)$. For further details, see Chen and Balakrishnan (1995).

The values of estimates, $-\log$ L, AIC, CAIC, BIC, HQCI are listed in Table 5 and $\mathrm{W}^{*}, \mathrm{~A}^{*}, \mathrm{~K}-\mathrm{S}$, p-values for all models are listed in Table 6 .

Table 5: MLEs of the parameters and some measures for the fitted models.

\begin{tabular}{|c|l|c|c|c|c|c|}
\hline Model & Parameters & $-\log L$ & AIC & CAIC & BIC & HQIC \\
\hline IE & $\hat{\lambda}=60.0975$ & 402.6718 & 807.3436 & 807.4007 & 809.6203 & 808.2499 \\
\hline IR & $\hat{\lambda}=46.7748$ & 406.7361 & 815.4722 & 815.5293 & 817.7489 & 816.3785 \\
\hline W & $\hat{\lambda}=0.0090, \hat{\beta}=1.3932$ & 397.1477 & 798.2954 & 798.4693 & 802.8487 & 800.1081 \\
\hline IW & $\hat{\lambda}=54.188, \hat{\beta}=1.4148$ & 395.6491 & 795.2982 & 795.4721 & 799.8515 & 797.1109 \\
\hline EIW & $\begin{array}{l}\hat{\alpha}=8.2725, \quad \hat{\lambda} \\
336.3775 \\
\hat{\beta}=0.6207\end{array}$ & 390.5000 & 787.0000 & 787.3529 & 793.8300 & 789.7190 \\
\hline IWL & $\hat{\alpha}=0.9002, \hat{\lambda}=719.78$ & 394.8100 & 795.6200 & 795.9729 & 802.4500 & 798.3590 \\
\hline
\end{tabular}




\begin{tabular}{|c|l|l|l|l|l|l|}
\hline & $\hat{\beta}=1.8558$ & & & & \\
\hline IWP & $\begin{array}{l}\hat{\alpha}=-13.8900, \hat{\lambda}=33.0030 \\
\hat{\beta}=0.5544\end{array}$ & 390.2600 & 786.5200 & 786.8729 & 793.3500 & 789.2390 \\
\hline IMOW & $\begin{array}{l}\hat{\alpha}=0.0162, \hat{\lambda}=14.1986 \\
\hat{\beta}=2.4845\end{array}$ & 389.7090 & 785.4180 & 785.7709 & 792.2480 & 788.1370 \\
\hline KW-MOI & $\begin{array}{l}\hat{\mathrm{a}}=68.1393, \hat{\mathrm{b}}=2.6258 \\
\hat{\alpha}=8.8727, \hat{\lambda}=0.1758\end{array}$ & 391.3500 & 790.7000 & 791.3000 & 799.8000 & 794.3000 \\
\hline EW-Fr & $\begin{array}{l}\hat{\mathrm{a}}=45.7326, \hat{\mathrm{b}}=8.2723 \\
\hat{\lambda}=0.7111, \hat{\beta}=0.6207\end{array}$ & 390.2500 & 788.5000 & 789.1000 & 797.6000 & 792.1000 \\
\hline EWP & $\begin{array}{l}0.2495 \\
\hat{\beta}=44.6070, \hat{\theta}=4.0840\end{array}$ & & & & & \\
\hline BFr & $\begin{array}{l}\hat{\alpha}=19.9786, \hat{\mathrm{b}}=20.1331 \\
\hat{\lambda}=24.5032, \hat{\beta}=0.3220\end{array}$ & 390.3000 & 788.6000 & 789.2000 & 797.7000 & 792.3000 \\
\hline$\hat{\lambda}=2.9633, \hat{\beta}=2.3122$ & 387.6737 & 782.9474 & 783.5444 & 792.0541 & 786.5728 \\
\hline
\end{tabular}

Table 6: Goodness-of-fit statistics for various models fitted to pig data.

\begin{tabular}{|c|c|c|c|c|}
\hline & & & & \\
Model & \multirow{2}{*}{$\mathrm{W}^{*}$} & $\mathrm{~A}^{*}$ & $\mathrm{~K}-\mathrm{S}$ & $\mathrm{p}$-value \\
& & & & \\
\hline IE & & & & \\
IR & 0.8322 & 4.5927 & 0.1847 & 0.0148 \\
W & 0.4325 & 6.4936 & 0.2508 & 0.0002 \\
IW & 0.2521 & 1.5017 & 1.1520 & 0.0718 \\
\hline
\end{tabular}




\begin{tabular}{|c|l|l|l|l|}
\hline \hline EIW & 0.1362 & 0.7546 & 0.1003 & 0.4642 \\
IWL & 0.1958 & 1.1447 & 0.1124 & 0.3516 \\
IWP & 0.1287 & 0.7188 & 0.0983 & 0.4899 \\
IMOW & 0.1030 & 0.6260 & 0.0900 & 0.6034 \\
Kw-MOIE & 0.1219 & 0.7404 & 0.1181 & 0.2674 \\
Kw-Fr & 0.1360 & 0.7544 & 0.1004 & 0.4623 \\
BFr & 0.1382 & 0.7631 & 0.0998 & 0.4418 \\
EWP & 0.1075 & 0.6158 & 0.0891 & 0.6176 \\
GIW & 0.0929 & 0.5464 & 0.0856 & 0.6668 \\
\hline
\end{tabular}

From the Table 5 and Table 6 , we can see that, GIW distribution has smallest $-\log \mathrm{L}, \mathrm{AIC}, \mathrm{CAIC}, \mathrm{BIC}, \mathrm{HQCI}, \mathrm{W}^{*}, \mathrm{~A}^{*}, \mathrm{~K}-\mathrm{S}$ values. Also the GIW distribution has highest $p$-value. Hence the new model, that is GIW distribution, yields a better fit than the other models for this data set.

The fitted density and the empirical cdf plot of the GIW distribution are presented in Figure 3. The figure indicates a satisfactory fit for the GIW distribution.

\section{Empirical Vs GIW distribution}
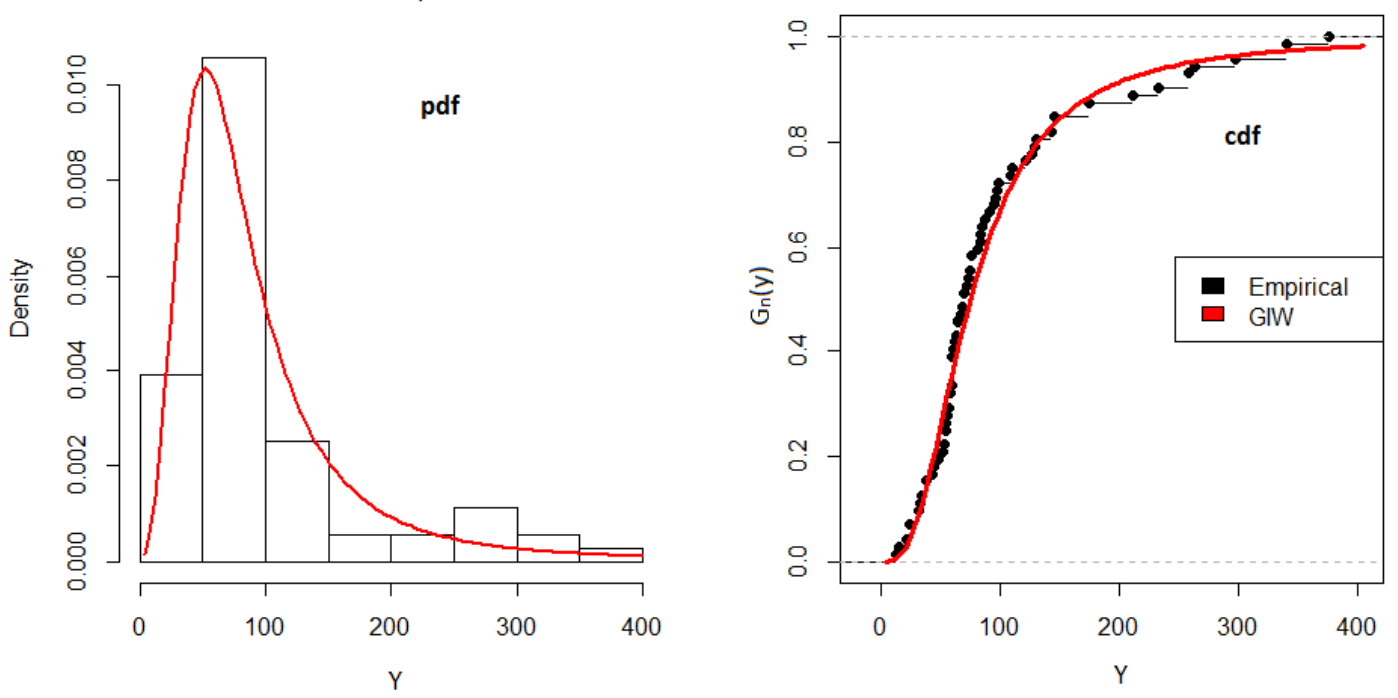

Figure 3: Plots of the estimated pdf and cdf of the GIW model for pig data.

To test the null hypothesis $\mathrm{H}_{0}$ : IMOW versus $\mathrm{H}_{1}$ : GIW or equivalently $\mathrm{H}_{0}: \theta=1$ versus $\mathrm{H}_{1}: \theta \neq 1$ we use likelihood ratio test statistic whose value is 4.4706(p-value $=0.0345$ ). As a result, the null model IMOW is rejected in favor of alternative model GIW at any level $>0.0345$.

\section{Acknowledgments}

The authors would like to thank the Editor and the reviewers for careful reading of the manuscript and the comments which greatly improved the 
presentation of the paper.

\section{References}

[1] Al-Fattah, A., El-Helbawy, A., Al-Dayian, G. (2017). Inverted Kumaraswamy distibution:properties and estimation. Pakistan Journal of Statistics, 33, 37-61.

[2] Bjerkedal, T. (1960). Acquisition of resistance in guinea pigs infected with different doses of virulent tubercle bacilli. American Journal of Epidemiology, 72, 130--148.

[3] Calabria, R., Pulcini, G. (1994). Bayes 2-sample prediction for the inverse Weibull distribution. Communications in Statistics- Theory and Methods, 23, 1811-1824.

[4] Chandra, S. (1977). On the mixtures of probability distributions. Scandinavian Journal of Statistics, 4, 105--112.

[5] Chakrabarty, J.B., Chowdhury, S. (2018). Compounded inverse Weibull distributions: Properties, inference and applications. Communications in StatisticsSimulation and Computation, 48, 2012- -2033.

[6] Chen, G., Balakrishnan, N. (1995). A general purpose approximate goodness-of-fit. Journal of Quality Technology, 27, 154--161.

[7] Cheng, R.C.H., Amin, N.A.K. (1983). Estimating parameters in continuous univariate distributions with a shifted origin. Journal of Royal Statistical Society. Series B, 45, 394--403.

[8] Cordeiro, G.M., Pescim, R.R., Ortega, E.M.M. (2012). The Kumaraswamy generalized half-normal distribution for skewed positive data. Journal of Data Science, 10, 195--224.

[9] Dubey, S.D (1970). Compound gamma, beta and F distribution. Metrika, 16, 27-31.

[10] Erto, P., Rapone, M. (1984). Non-informative and practical Bayesian confidence bounds for reliable life in the Weibull model. Reliability Engineering, 7, 181-191.

[11] Folks, J., Chhikara, R. (1978). The inverse Gaussian distribution and its statistical application-a review. Journal of Royal Statistical Society Series B, 40, 263-289. 
[12] Gharib, M., Mohammed, B.I., Aghel, W.E.R. (2017). Marshall-Olkin extended inverse Pareto distribution and its application. International Journal of Statistics and Probability, 6, 71-84.

[13] Gupta, P. L., Gupta, R.C. (1983). On the moments of residual life in reliability and some characterization results. Communications in StatisticsTheory and Methods, 12, 449--461.

[14] Hassan, A.S., Nassar, S.G. (2018). The inverse Weibull generator of distributions: Properties and applications. Journal of Data Science, 16, 723--742.

[15] Jayakumar, K., Sankaran, K.K. (2018). Discrete Linnik Weibull distribution. Communications in Statistics - Simulation and Computation, 48, 3092-3117.

[16] Khan, M.S., Pasha, G.R., Pasha, A.H. (2008). Theoretical analysis of inverse Weibull distribution. WSEAS Transactions on Mathematics, 7, 30--38.

[17] Kundu, D., Howlader, H. (2010). Bayesian inference and prediction of the inverse Weibull distribution for Type-II censored data. Computational Statistics and Data Analysis, 54, 1547--1558.

[18] Kundu, D., Nanda, A.K. (2010). Some reliability properties of the inactivity time. Communications in Statistics - Theory and Methods, 39, 899--911.

[19] Marshall, A.W., Olkin, I. (1997). A new method for adding a parameter to a family of distributions with application to the exponential and Weibull families. Biometrika, 84, 641--652.

[20] Murthy, D.P., Xie, M., Jiang, R. (2004). Weibull Models (vol. 505). John Wiley and Sons, New Jersey.

[21] Nadarajah, S., Jayakumar, K., Ristić, M.M. (2013). A new family of lifetime models. Journal of Statistical Computation and Simulation, 83, 1389--1404.

[22] Nanda, A.K., Sing, H., Misra, N., Paul, P. (2003). Reliability properties of reversed residual lifetime. Communications in Statistics - Theory and Methods, 32, 2031--2042.

[23] Okasha, H.M., El-Ba, A.H., Tarabia, A.M.K., Basheer, A.M. (2017). Extended inverse Weibull distribution with reliability application. Journal of 
the Egyptian Mathematical Society, 25, 343--339.

[24] Pillai, R.N. (1990). On Mittag-Leffler functions and related distributions. Annals of the Institute of Statistical Mathematics, 42, 157-161.

[25] Pillai, R.N., Jayakumar, K. (1995). Discrete Mittag-Leffler distributions. Statistics and Probability Letters, 23, 271-274.

[26] Prakash, G. (2012). Inverted exponential distribution under a Bayesian view point. Journal of Modern Applied Statistical Methods, 11, 190-202.

[27] Rana, M.U., Muhammed Ahsan ul Haq. (2018). The Marshall-Olkin extended inverted Kumaraswamy distribution: Theory and application. Journal of King Saud University, https://doi.org/10.1016/j.jksus.2018.05.021.

[28] Sankaran, K.K., Jayakumar, K. (2016). A new extended uniform distribution. International Journal of Statistical Distributions and Applications, 2, 35--41.

[29] Shafiei, S., Darijani, S., Saboori, H. (2016). Inverse Weibull power series distributions: properties and applications. Journal of Statistical Computation and Simulation, 86, 1069--1094.

[30] Shaked, M., Shanthikumar, J.G. (2007). Stochastic Orders. Springer, New York, 2007.

[31] Sharma, V.K., Singh, S.K., Singh, U., Agiwal, V. (2015). The inverse Lindley distribution: a stress-strength reliability model with application to head and neck cancer data. Journal Industrial and Production Engineering, 32, 162-173.

[32] Tahir, M.H., Nadarajah, S. (2015). Parameter induction in continuous univariate distributions: Well-established G families. Annals of the Brazilian Academy of Sciences, 87, 539--568.

[33] Voda, V.G. (1972). On the inverse Rayleigh distributed random variable. Reports of Statistical Application Research, 19, 13-21. 